\title{
Article
}

\section{Standardization of complex biologically derived spectrochemical datasets}

Morais, Camilo L M, Paraskevaidi, Maria, Cui, Li, Fullwood, Nigel J, Isabelle, Martin, Lima, Kássio M G, Martin-Hirsch, Pierre L, Sreedhar, Hari, Trevisan, Júlio, Walsh, Michael J, Zhang, Dayi, Zhu, Yong-Guan and Martin, Francis L

Available at http://clok.uclan.ac.uk/28305/

Morais, Camilo L M ORCID: 0000-0003-2573-787X, Paraskevaidi, Maria, Cui, Li, Fullwood, Nigel J, Isabelle, Martin, Lima, Kássio M G, Martin-Hirsch, Pierre L, Sreedhar, Hari, Trevisan, Júlio et al (2019) Standardization of complex biologically derived spectrochemical datasets. Nature Protocols, 14 . pp. 1546-1577. ISSN 1754-2189

It is advisable to refer to the publisher's version if you intend to cite from the work. http://dx.doi.org/10.1038/s41596-019-0150-x

For more information about UCLan's research in this area go to http://www.uclan.ac.uk/researchgroups/ and search for < name of research Group>.

For information about Research generally at UCLan please go to

http://www.uclan.ac.uk/research/

All outputs in CLoK are protected by Intellectual Property Rights law, including Copyright law. Copyright, IPR and Moral Rights for the works on this site are retained by the individual authors and/or other copyright owners. Terms and conditions for use of this material are defined in the policies page. 


\section{Standardization of complex biologically-derived spectrochemical datasets}

Camilo L.M. Morais ${ }^{\mathrm{a},{ }^{*}, 1}$, Maria Paraskevaidi ${ }^{\mathrm{a},{ }^{*}, 1}$, Li Cui ${ }^{\mathrm{d}}$, Nigel J Fullwood ${ }^{\mathrm{b}}$, Martin Isabelle ${ }^{\mathrm{c}}$, Kássio M.G. Lima ${ }^{\mathrm{e}}$, Pierre L. Martin-Hirsch ${ }^{\mathrm{f}}$, Hari Sreedhar $^{\mathrm{h}}$, Júlio Trevisan ${ }^{\mathrm{g}}$, Michael J Walsh ${ }^{\mathrm{h}}$, Dayi Zhangi, Yong-Guan Zhu ${ }^{\mathrm{d}}$, Francis L. Martin ${ }^{\mathrm{a}, 1}$

${ }^{a}$ School of Pharmacy and Biomedical Sciences, University of Central Lancashire, Preston PRI 2HE, UK; ${ }^{b}$ Division of Biomedical and Life Sciences, Faculty of Health and Medicine, University of Lancaster, Lancaster LA1 4YQ, UK; ${ }^{c}$ Spectroscopy Products Division Renishaw plc, New Mills, Wotton-under-Edge, Gloucestershire GL12 8JR, UK; ${ }^{d}$ Key Lab of Urban Environment and Health, Institute of Urban Environment, Chinese Academy of Sciences, Xiamen 361021, China; ${ }^{e}$ Institute of Chemistry, Biological Chemistry and Chemometrics, Federal University of Rio Grande do Norte, Natal 59072-970, Brazil; ${ }^{f}$ Department of Obstetrics and Gynaecology, Lancashire Teaching Hospitals NHS Foundation, Preston PR2 9HT, UK; ${ }^{g}$ Institute of Astronomy, Geophysics and Atmospheric Sciences, University of São Paulo, Cidade Universitária, R. do Matão, 1226 Butantã, São Paulo - SP, 05508-090, Brazil; ${ }^{h}$ Department of Pathology, University of Illinois at Chicago, Chicago, Illinois, USA; 'iSchool of Environment, Tsinghua University, Beijing 100084, China

${ }^{1}$ To whom correspondence should be addressed: Email: cdlmedeiros-de-morai@uclan.ac.uk; Email: mparaskevaidi@uclan.ac.uk; Email: flmartin@uclan.ac.uk; Tel: +44 (0) 1772896482

*Contributed equally 


\section{Abstract}

Spectroscopic techniques, such as Fourier-transform infrared (FTIR) spectroscopy, are used to study the interaction of light with biological materials. This interaction forms the basis of many analytical assays used in disease screening and diagnosis, microbiological studies, forensic and environmental investigations. Advantages of spectrochemical analysis are its low cost, minimal sample preparation, non-destructive nature and substantially accurate results. However, there is now an urgent need for repetition and validation of these methods in large-scale studies and across different research groups, which would bring the method closer to clinical and/or industrial implementation. In order for this to succeed, it is important to understand and reduce the effect of random spectral alterations caused by inter-individual, inter-instrument and/or inter-laboratory variations, such as variations in air humidity and $\mathrm{CO}_{2}$ levels, and the aging of instrumental parts. Thus, it is evident that spectral standardization is crucial for the widespread adoption of these spectrochemical technologies. By using calibration transfer procedures, where the spectral response of a secondary instrument is standardized to resemble the spectral response of a primary instrument, different sources of variations can be normalized into a single model using computational-based methods, such as direct standardization (DS) and piecewise direct standardization (PDS); therefore, measurements performed under different conditions can generate the same result, eliminating the need for a full recalibration. In this paper, we have constructed a protocol for model standardization using different transfer technologies described for FTIR spectrochemical applications. This is a critical step towards the construction of a practical spectrochemical analysis model for daily routine analysis, where uncertain and random variations are present. 
Introduction

Vibrational spectroscopy has shown great promise as an analytical tool for the investigation of numerous sample types with wide applications in diverse sectors, such as biomedicine, pharmaceutics or environmental sciences ${ }^{1-5}$. Fourier-transform infrared (FTIR) spectroscopy is one of the preferred techniques for identification of biomolecules through the study of their characteristic vibrational movements. Another commonly used approach is Raman spectroscopy, which provides complementary spectral information to IR. Raman spectroscopy exploits the inelastic scattering of light whereas IR studies light absorption. Both methods have their benefits and drawbacks. A limitation of IR, for instance, is that water generates undesired peaks at the region of interest, which can mask important biological information, and therefore extra sample preparation and/or spectral processing is necessary. On the contrary, Raman spectroscopy has an inherently weak signal and fluorescence interference, which can, however, be addressed by optimizing the experimental settings or by applying enhancement techniques to increase the Raman signal. For the purposes of this protocol we have used FTIR spectroscopy to demonstrate our standardization model.

Using chemometric approaches, the system is trained to recognize unique spectral features within a sample, so that when unknown samples are introduced an accurate classification is feasible. Alterations in the measurement parameters could interfere with the spectral signature and produce random variations. Therefore, a crucial step is spectral correction, or standardization, which would provide comparable results and allow system transferability. The idea is that nonbiological variations, such as those arising from different users, locations or instruments, will no longer affect the classification result; therefore any collected data could be imported into a central database and handled for further exploration or diagnostic purposes. Several groups and companies 
worldwide are developing spectrochemical approaches for diagnosis, discrimination and monitoring of diseases, as well as for other uses. Combination of multiple datasets would facilitate the conduction of large-scale studies which are still lacking in the field of bio-spectroscopy.

Sensor-based technologies

Sensor-based technologies are an integral part of daily life ranging from locating sensorbased technology, such as global positioning system $(\mathrm{GPS})^{6}$, to image biosensors, such as X-rays ${ }^{7-}$ ${ }^{10}$ and $\gamma$-rays ${ }^{11-13}$, which are used extensively for medical applications. Other powerful approaches that make use of sensor-based technologies toward medical disease examination and diagnostics include circular dichroism (CD) spectroscopy ${ }^{14-17}$, ultraviolet (UV) or visible spectroscopy ${ }^{18,19}$, fluorescence ${ }^{20-24}$, nuclear magnetic resonance (NMR) spectroscopy ${ }^{25-29}$ and ultrasound (US) ${ }^{7,30-}$ 33.

Over the last two decades, optical biosensors employing vibrational spectroscopy, particularly IR spectroscopy, have seen tremendous progress in biomedical and biological research. A number of studies using the above-mentioned methods have focused on cancer investigation with malignancies such as brain $^{34-37}$, breast ${ }^{38-40}$, oesophagus ${ }^{41,42}$, skin $^{43-47}$,

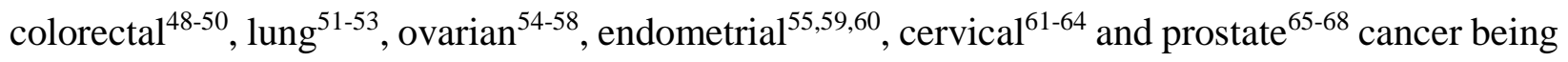
some of them. Non-cancerous diseases have also been examined, namely neurodegenerative disorders $^{69-72}$, HIV/AIDS $^{73}$, diabetes ${ }^{74-76}$, rheumatoid arthritis ${ }^{77,78}$, cardiovascular diseases $^{79,80}$, malaria $^{81-83}$, alkaptonuria ${ }^{84}$, cystic fibrosis ${ }^{85}$, thalassemia ${ }^{86}$, prenatal disorders ${ }^{87,88}$, macular degeneration ${ }^{89,90}$, atherosclerosis ${ }^{80,91}$ and osteoarthritis ${ }^{92-94}$. 


\section{Limitations}

Spectrochemical approaches are advantageous when compared with traditional molecular methods as they provide a holistic status of the sample under interrogation, thus generating typical spectral regions widely known as "fingerprint regions". These methods have also been shown to be rapid, inexpensive and non-destructive while they also improve diagnostic performance and eliminate subjective diagnosis (e.g., histopathological diagnosis), where inter- and intra-observer variability are present ${ }^{95}$. However, like any other analytical method, vibrational spectroscopy also comes with some limitations. For instance, prior to FTIR studies, optimization of instrumental settings, sample preparation and operation mode also needs to be conducted in order to improve the spectral quality and molecular sensitivity ${ }^{4,96,97}$. Overall, the above-mentioned barriers can be overcome after careful consideration of the experimental design.

A considerable limitation that is yet under-investigated in the field of spectrochemical techniques is associated with the difficulties entailed in data conformation and system standardization. Currently, there are multiple pilot studies showing promising results but an approach towards standardization for biological applications is lacking. Random variation between studies can originate from differences in instrumentation, operators, and environmental conditions, such as room temperature and humidity.

The main objective of this article is to present a protocol for model standardization which can be applied in FTIR spectrochemical techniques to rule out the chance of random spectral alterations. Inter-individual, inter-instrument, inter-sample and/or inter-laboratory variations can be a source of unwanted, non-biological alterations, thus leading to incorrect conclusions. However, for a method to become reliable and clinically translatable, it is important that measurements performed under different conditions generate comparable results. The aim of the 
spectral standardization model presented here is to expedite multi-centre studies with large numbers of samples; this would bring these spectrochemical techniques closer to clinical implementation and facilitate life-changing decisions. We describe a protocol that has four main components: (i) sample preparation, (ii) spectral acquisition, (iii) data pre-processing and (iv) model standardization. The current protocol has an in-depth insight obtained from cross-laboratory collaborations with leading experts in the field. This article offers a step-by-step procedure, which can be implemented by a non-specialist in spectrochemical studies. For further information about instrumental and software options, spectral acquisition steps and data analysis for a range of different analytical systems the reader is directed towards additional protocols ${ }^{4,98-105}$.

\section{Applications}

Spectrochemical approaches, in combination with computational analysis, have been proven to be effective for biomedical research through facilitating the diagnosis, classification, prognosis, treatment stratification and modulation or monitoring of a disease and treatment. However, these techniques are widely applicable to other fields as well, namely food industry ${ }^{106-}$ ${ }^{109}$, toxicology $y^{2,110-112}$, microbiology ${ }^{113-118}$, forensics ${ }^{119-123}$, pharmacy ${ }^{2,3,124}$, environmental and plant science ${ }^{125-127}$, as well as defence and security ${ }^{128-130}$. Applications of standardization algorithms vary according to the spectral technique and sample matrix studied, and have been mostly applied to Raman and Fourier-transform near-infrared (FT-NIR) spectroscopy. Table 1 summarizes some standardization applications. 
1 Table 1. Examples of applications involving standardization techniques.

\begin{tabular}{|c|c|c|c|}
\hline Sample matrix & Spectroscopic technique & Aim & Ref. \\
\hline \multirow[t]{2}{*}{ Tissue } & Raman & $\begin{array}{l}\text { Standardization of various perturbations on Raman spectra for diagnosis of breast cancer based on snap frozen } \\
\text { tissues }\end{array}$ & 131 \\
\hline & Raman & $\begin{array}{l}\text { Standardization of spectra acquired in } 3 \text { different sites for analysing oesophageal samples based on snap frozen } \\
\text { tissues }\end{array}$ & 132 \\
\hline Cells & Raman & $\begin{array}{l}\text { Standardization of spectra acquired with } 4 \text { different instruments for classification of three different cultured spore } \\
\text { species }\end{array}$ & 133 \\
\hline \multirow[t]{2}{*}{ Biofluids } & FT-NIR & $\begin{array}{l}\text { Standardization of spectra acquired with } 3 \text { different instruments for measuring haematocrit in the blood of } \\
\text { grazing cattle }\end{array}$ & 134 \\
\hline & LC-MS & $\begin{array}{l}\text { Standardization of spectra acquired with } 2 \text { different instruments for mapping rendition times and matching } \\
\text { metabolite features of subjects diagnosed with small cell lung cancer based on blood serum and plasma samples } \\
\text { analysis }\end{array}$ & 135 \\
\hline \multirow[t]{3}{*}{ Pharmaceutical materials } & Raman & $\begin{array}{l}\text { Standardization of spectra acquired with } 5 \text { different instruments for analysing various pharmaceutical excipients, } \\
\text { active pharmaceutical ingredients (APIs) and common contaminants }\end{array}$ & 136 \\
\hline & FT-NIR & $\begin{array}{l}\text { Standardization of spectra acquired with } 2 \text { different instruments for simultaneous determination of rifampicin } \\
\text { and isoniazid in pharmaceutical formulations }\end{array}$ & 137 \\
\hline & FT-NIR & $\begin{array}{l}\text { Standardization of spectra acquired with } 2 \text { different instruments for predicting content of } 654 \text { pharmaceutical } \\
\text { tablets }\end{array}$ & 138 \\
\hline \multirow[t]{5}{*}{ Food } & FT-NIR & Standardization of spectra acquired with 3 different instruments for predicting parameters in corn samples & 138 \\
\hline & FT-NIR & Standardization of spectra acquired with 2 different instruments for predicting vitamin $\mathrm{C}$ in navel orange & 140 \\
\hline & FT-NIR & $\begin{array}{l}\text { Standardization of spectra recorded in } 4 \text { different labs for determining moisture, proteins and oil content in soy } \\
\text { seeds }\end{array}$ & 141 \\
\hline & FT-NIR & $\begin{array}{l}\text { Standardization of spectra acquired by a benchtop and portable instrument for determining total soluble solid } \\
\text { contents in single grape berry }\end{array}$ & 142 \\
\hline & UV-Vis & Standardization of visible spectra acquired with 3 different instruments for measuring pH of Sala mango & 143 \\
\hline \multirow[t]{3}{*}{ Plant } & FT-NIR & $\begin{array}{l}\text { Standardization of spectra acquired with } 2 \text { different instruments for predicting baicalin contents in radix } \\
\text { scutellariae samples }\end{array}$ & 139 \\
\hline & FT-NIR & $\begin{array}{l}\text { Standardization of spectra acquired by } 2 \text { different instruments and in three physical states (powder, filament and } \\
\text { intact leaf) for determining total sugars, reducing sugars and nicotine in tobacco leaf samples }\end{array}$ & 144 \\
\hline & NMR & Standardization of spectra acquired with 3 different instruments for authenticity control of sunflower lecithin & 145 \\
\hline Cosmetic & CD spectroscopy & $\begin{array}{l}\text { Standardization of spectra acquired between standard and real-world samples for determining } \mathrm{Pb}^{2+} \text { in cosmetic } \\
\text { samples }\end{array}$ & 146 \\
\hline Inorganic substances & FT-IR & Standardization of interferogram spectra acquired with 2 instruments for classifying acetone and $\mathrm{SF}_{6}$ samples & 147 \\
\hline Fuel & FT-IR & Standardization of spectra acquired with 2 different instruments for predicting density of crude oil samples & 148 \\
\hline
\end{tabular}


Model transferability

Transferability models have been previously developed, however this is still an underinvestigated field, especially for biomedical applications. These models use computer-based methods to standardize spectral data generated across different experimental settings (e.g., different instruments, operators or laboratories). An inclusive standardization protocol that could be implemented in a range of different spectrochemical approaches is of great need. Differences are present even between identical instruments; for instance, changes in signal intensity caused by replacement, alignment or ageing of optical and spectrometer components, natural variations in optics and detectors construction, changes in measurement conditions (temperature and humidity), changes in physical constitution of the sample (particle size and surface texture) and operator discrepancies could all lead to wavenumber shifts and artefacts in the spectra. In all of these cases, prediction errors of the estimated group categories (e.g., whether the sample is classified as healthy or cancerous) can become very large, especially when the whole spectrum is used in the model. Standardization techniques aim to generate a uniform spectral response under differing conditions, ensuring the interchangeability of results obtained in different situations, without having to perform a full calibration for each situation.

Previous standardization methods include the use of simple slope and bias correction $^{149,150}$, direct standardization (DS) ${ }^{151-155}$, piecewise direct standardization $(\mathrm{PDS})^{149,156-158}$, piecewise linear discriminant analysis $(\mathrm{PLDA})^{147}$, guided model reoptimization $(\mathrm{GMR})^{158}$, back-propagation neural network $(\mathrm{BNN})^{147}$, generalized least squares weighting $(\mathrm{GLSW})^{159}$, model updating (MU) ${ }^{160,161}$, orthogonal signal correction $(\mathrm{OSC})^{162,163}$, orthogonal projections to latent structures (OPLS) ${ }^{148}$, wavelet hybrid direct standardization (WHDS) ${ }^{157}$, maximum likelihood PCA (MLPCA) ${ }^{164}$, Shenk and Westerhaus method $(\mathrm{SW})^{165,166}$, positive matrix factorization (PMF) ${ }^{167,168}$, artificial neural networks (ANN) drift correction ${ }^{169}$, transfer via extreme learning machine auto-encoder method (TEAM) ${ }^{170}$, 
calibration transfer based on the maximum margin criterion (CTMMC) ${ }^{171}$, calibration transfer based on canonical correlation analysis $(\mathrm{CTCCA})^{172}$ and calibration methods, such as wavenumber offset correction, instrument response correction and baseline correction ${ }^{132}$. In this protocol, we use direct standardization (DS) and piecewise direct standardization (PDS), because they are the most common methods for spectral standardization.

Direct standardization. DS is one of the most used methods for data standardization. It was initially proposed to correct relatively large spectral differences between data collected from the same sample measured by two different instruments ${ }^{149}$. In DS, the entire spectrum from a new secondary response (e.g., a different instrument) is transformed to resemble the spectrum

37 from the primary source (e.g., original instrument $)^{151}$. This is performed based on a linear relationship between the data acquired under different circumstances ${ }^{160}$ :

where $\mathbf{S}_{1}$ represents the data acquired for the primary response; $\mathbf{S}_{2}$ represents the data acquired for the secondary response; and $\mathbf{F}$ is the transformation matrix that maintains the relationship between $\mathbf{S}_{1}$ and $\mathbf{S}_{2}$.

where $\mathbf{S}_{2}^{+}$is the pseudo-inverse of $\mathbf{S}_{2}$, calculated by: in which $\mathrm{T}$ stands for the matrix transpose operation.

Then, when samples are measured under the secondary system, the signals generated $\mathbf{X}$ are transformed to resemble the primary system response by ${ }^{160}$ : 
51 where $\widehat{\mathbf{X}}$ is the standardized response for $\mathbf{X}$.

Problems related to different background information between instruments can affect the standardization procedure. To correct for this, the standardization process is usually adapted with the background correction method ${ }^{173}$, in which the transformation matrix described in Eq. 02 is calculated with a background correction factor $\left(\mathbf{F}_{\mathrm{b}}\right)$ and an additive background correction vector $\mathbf{b}_{\mathrm{s}}$ as follows:

$57 \quad \mathbf{S}_{1}=\mathbf{S}_{2} \mathbf{F}_{\mathrm{b}}+\mathbf{1} \mathbf{b}_{\mathrm{s}}^{\mathrm{T}}$

where $\mathbf{1}$ is an all-ones vector and $\mathbf{b}_{\mathbf{s}}$ is obtained by:

$\mathbf{b}_{\mathrm{s}}=\mathbf{s}_{1 \mathrm{~m}}-\mathbf{F}_{\mathrm{b}}^{\mathrm{T}} \mathbf{s}_{2 \mathrm{~m}}$

in which $\mathbf{s}_{1 \mathrm{~m}}$ is the mean vector of $\mathbf{S}_{1}$ and $\mathbf{S}_{2 \mathrm{~m}}$ is the mean vector of $\mathbf{S}_{2}$.

One of the key steps for DS is the selection of the number of samples to transfer (called “transfer samples"). These are samples' spectra from the primary system $\left(\mathbf{S}_{1}\right)$ that will be used to transform the signal obtained using the secondary system $\left(\mathbf{S}_{2}\right)$. The transfer samples are obtained from a same cohort of samples (e.g., plasma samples) measured in the two instruments (primary and secondary systems). Usually, the procedure for selecting transfer samples is based on sample selection techniques, such as Kennard-Stone (KS) algorithm ${ }^{174}$ or leverage ${ }^{149}$. Subsequently, the number of transfer samples is evaluated using a validation set through an arbitrary cost function. For quantification applications, a common cost function is the rootmean-square error of prediction, while for classification one can use the misclassification rate.

A disadvantage of DS is that each transformed variable is calculated using the whole spectrum, which carries a high risk of overfitting. The estimation of $\mathbf{F}$ in Eq. (02) is an ill- 
conditioned problem, because the number of variables (e.g., wavenumber) may be much larger than the number of standard samples.

Piecewise direct standardization. PDS is another standardization procedure commonly employed for system transferability. It is based on DS, however it uses windows (e.g., wavenumber portions) to make the standardization process more suitable for smaller regions of the data. When compared to DS, PDS is calculated by using the transformation matrix $\mathbf{F}$ with most of its off-diagonal elements set to zero ${ }^{149}$. With this, PDS fits minor spectral modifications not covered by DS. PDS is the technique of preference for correcting smaller spectral variations, such as small wavelengths shift, intensity variations, and bands enlargement and reduction ${ }^{149}$. In addition, an advantage of PDS compared to DS is that the local rank of each window will be smaller than the rank of the whole data matrix, which means that the number of standard samples can be smaller, and indeed good results have been obtained with very few samples.

One disadvantage of PDS is the need of an additional optimization process, because in addition to the number of transfer samples, PDS also needs a window size optimization, which might lead to a risk of overfitting. In this protocol, window size optimization is made using a cost function expressed as the misclassification rate calculated for each window size tested, being evaluated using a validation set where the window with smaller misclassification is selected for final model construction.

\section{Experimental Design}

Any study using vibrational spectroscopy, follows these general steps: careful experimental design, protocol optimisation and development of experimental procedure document, sample collection and preparation, spectral collection, pre-processing of the derived information and lastly the use of chemometrics for exploratory, classification and 
standardization purposes. FTIR spectroscopy is described in more detail in this study, however, the standardization protocol described here can be adapted to a range of techniques, including attenuated total reflection (ATR-FTIR), transmission and transflection FTIR, near-IR (NIR), UV-visible, NMR spectroscopy and mass spectrometry (MS). Nevertheless, intrinsic features of each technique should be taken into consideration before standardization and the protocol may change depending on the application of interest.

A number of biological samples can be analyzed with the above-mentioned analytical methods such as tissues, cytological materials or biological fluids. Sample type and preparation may differ depending on the technique that is employed each time. For instance, IR spectroscopy is limited by water interference at the fingerprint region that can mask the signal of the analyte close to the water peak. This could be addressed with an extra step of sample drying, in contrast to Raman spectroscopy, for example, where water does not generate signal in this region.

Typical steps for sample preparation, acquisition of spectra and data pre-processing are briefly presented here. However, the main focus of this protocol is placed on the calibration transfer and standardization procedures. Readers are directed to additional literature for more detailed information regarding sample format and preparation ${ }^{4,98-100,105,175-177}$, suitability of substrates $^{4,99}$, instrumentation settings $\mathrm{s}^{4,98,99,105,175,177,178}$ or available software packages (Table 2) and manufacturers ${ }^{4,99}$.

Table 2. Software packages for data standardization.

\begin{tabular}{llll}
\hline Software & Website & Description & Availability \\
\hline PLS_Toolbox & http://www.eigenvector.com/ & MATLAB toolbox for chemometric Commercial \\
& & analysis. Contains standardization \\
& routines using DS, PDS, double \\
& window PDS, spectral subspace \\
& transformation, GLSW, OSC, and \\
& alignment of matrices. \\
Unscrambler®X & $\underline{\text { http://www.camo.com/ }}$ & $\begin{array}{l}\text { Software for multivariate data Commercial } \\
\text { analysis and design of experiments. }\end{array}$
\end{tabular}




\begin{tabular}{|c|c|c|c|}
\hline & & $\begin{array}{l}\text { Contains standardization routines } \\
\text { using interpolation, bias and slope } \\
\text { correction, and PDS. }\end{array}$ & \\
\hline OPUS & $\underline{\text { https://www.bruker.com/ }}$ & $\begin{array}{l}\text { Spectral acquisition software with } \\
\text { data processing features. Contains a } \\
\text { standardization routine using PDS. }\end{array}$ & Commercial \\
\hline Pirouette $\AA$ & https://infometrix.com/ & $\begin{array}{l}\text { Chemometrics modelling software. } \\
\text { Contains standardization routines } \\
\text { using DS and PDS. }\end{array}$ & Commercial \\
\hline
\end{tabular}

Experimental design: sampling

118 Sample preparation. Biological samples have been studied extensively with spectrochemical techniques for disease research. Tissue specimens can be analysed fresh, snap-frozen or formalin-fixed, paraffin-embedded (FFPE). Fresh or snap-frozen histology sections are preferable as they are devoid of contaminants whereas FFPE treatment contributes to characteristic peaks, hindering the biological information. FFPE tissues can be deparaffinized either by chemical methods (e.g., incubation in xylene, hexane or Histo-Clear solutions $)^{4}$, which can alter tissue structures and be inefficient for the complete wax removal ${ }^{179}$, or by applying chemometrics (e.g., digital dewaxing $)^{180,181}$, which keeps the tissue intact but might introduce artefacts due to over- or under-estimation of the wax contribution ${ }^{179}$.

Fixatives, such as ethanol, methanol or formalin, are often used for the preservation of cytological material, also generating strong peaks and interfering with the spectra; thus, a washing step is crucial before spectroscopic interrogation. Fixation in tissue or cells for preservation purposes generates protein cross-linking which can cause changes in the spectra, especially on the Amide I peak ${ }^{182}$. Alternatively, cells can be studied live after washing from residual medium. the biofluids that have been previously used in spectroscopic studies include blood (whole blood, plasma or serum), urine, sputum, saliva, tears, cerebrospinal fluid (CSF), synovial fluid, ascitic fluid or amniotic fluid ${ }^{183-185}$. An initial centrifugation step should precede analysis in 
cases where the cells present in these fluids are not the focus of the study; the supernatant could then be kept for further analysis. In blood-based studies, the user should also consider the anticoagulant of preference (e.g., EDTA, citrate or heparin) as it could generate unwanted spectral peaks ${ }^{186-188}$. Careful planning of experiments as well as consistence throughout a study are of great importance for the generation of robust results. Care should be taken to generate samples that are stable, since the spectral differences between the data collected under different situations (e.g., different instruments or temperature) should be directly related to the difference between the systems and not a change caused by chemical or physical degradation of the samples. Optimal sample thickness, suitability of substrates and sample formats can differ from one analytical technique to another and thus the user should decide and tailor these according to the study's objective (a list with appropriate substrates is given in the Materials-Equipment section). Another consideration is the number of freeze-thaw cycles and long-term storage as these could compromise the integrity of the samples ${ }^{186,189}$. Preferably, FFPE tissue samples should be analysed after thorough dewaxing and freeze-thaw cycles or long-term storage avoided since these could result in many confounding factors for analysis.

Spectral acquisition. Depending on the study's objective, FTIR spectral information can be collected using either point spectra or imaging.

FTIR spectra can be collected in different operational modes, namely ATR-FTIR, transmission or transflection. Instrument parameters such as resolution, aperture size, interferometer mirror velocity and co-additions have to be optimised before acquisition of spectra to achieve high $\mathrm{SNR}^{4,98}$. Metal surfaces can also be used to increase the IR signal in a technique known as surface-enhanced IR absorption (SEIRA) ${ }^{190,191}$. As water interference can mask biological information in IR spectra, the user can purge the spectrometer with dry air or nitrogen gas to reduce the internal humidity of the instrument, or use computational analysis to remove the water signature. In addition, samples should be dried until all water content 
evaporates; however, drying of a sample is not without consequences, since chemical changes may occur such as loss of volatile compounds. A background sample is collected regularly to account for any changes in the atmospheric or instrument conditions.

For analysing homogenous samples (e.g., biofluids), measurements can be performed by acquiring spectra on different regions of the centre of a drop and across its borders. In transmission measurements, the sample can be measured raw or diluted. Usually, 10 spectra are collected per sample. A higher number of spectral replicas can be performed to decrease the standard-deviation (SD) between measurements, since the SD is proportion to $1 / \sqrt{n}$, where $n$ is the number of replicas. For heterogeneously distributed samples (e.g., tissues), spectra should be acquired covering the sample surface as uniformly as possible, to ensure that all sources of variation in the samples are stored in the spectral data. Samples replicas are also recommended at least as triplicates. For precision estimation, at least six replicates at three levels should be performed. The minimum number of samples for analysis can be estimated using a power test at an $80 \%$ power $^{192}$. Further details regarding sampling methodologies for analysing biological materials using FT-IR spectroscopy can be found in our previous protocols ${ }^{4,98}$.

\section{Experimental design: data quality evaluation}

Before processing, the data can be assessed to identify presence of anomalous behaviours or biased patterns. This can be made initially by visual inspection (e.g., identification of very anomalous spectra) followed by Hotelling $\mathrm{T}^{2}$ versus $\mathrm{Q}$ residuals charts using only the mean-centred spectra. PCA residuals ${ }^{193}$ can be explored to identify biased patterns, in which heteroscedastic distributions are signs of biased experimental measurements; while homoscedastic distributions are associated with good sampling. SNR can be estimated by dividing the power $(P)$ of signal by the power of noise, that is $\mathrm{SNR}=P_{\text {signal }} / P_{\text {noise }}=$ 
$186\left(A_{\text {signal }} / A_{\text {noise }}\right)^{2}$, where $A$ is the amplitude; or by the inverse of the coefficient of variation, 187 when only non-negative variables are measured. Collinearity can be evaluated by calculation 188 of the condition number, which is a matrix calculation that measures how sensitive the result is to perturbations in the input data (i.e., spectra) and to roundoff errors made during the solution process. This value is naturally high for spectral data (high collinearity).

\section{Experimental design: pre-processing}

Data pre-processing is used to maximise the SNR. This process is fundamental for correcting physical interferences, such as light scattering, different sample thickness, different optical paths and instrumental noise. Therefore, the pre-processing step has fundamental importance to highlight the signal of interest, reduce interferences and possibly correct anomalous samples.

For standardization applications, the pre-processing step is also important for reducing differences between the different systems that are used. Before any additional pre-processing, the spectrum should be trunctated to the biofingerprint region (e.g., $900-1800 \mathrm{~cm}^{-1}$ ) before analysis. This region contains the main absorptions from biochemical compounds and it suffers only minor effects of environmental variability, such as air humidity (free $v \mathrm{O}-\mathrm{H}=3650-3600$ $\mathrm{cm}^{-1}$, hydrogen-bonded $\left.v \mathrm{O}-\mathrm{H}=3400-3300 \mathrm{~cm}^{-1}\right)$ and air $\mathrm{CO}_{2}\left(v_{\mathrm{s}} \mathrm{CO}_{2}=2350 \mathrm{~cm}^{-1}\right)^{194}$. Table $\mathbf{3}$ summarizes the main pre-processing techniques for correcting noise in biologically-derived datasets. 
205 Table 3. Main pre-processing used for biologically-derived datasets.

\begin{tabular}{|c|c|c|c|c|c|}
\hline Pre-processing & Interfering & Technique & Advantage & Disadvantage & Optimization \\
\hline $\begin{array}{l}\text { Savitzky-Golay } \\
\text { smoothing }{ }^{195}\end{array}$ & Instrumental noise & $\begin{array}{l}\text { ATR-FTIR, FTIR, } \\
\text { NIR, Raman, NMR, } \\
\text { UV-Vis }\end{array}$ & $\begin{array}{c}\text { Corrects spectral noise } \\
\text { without changing the shape } \\
\text { of data significantly }\end{array}$ & $\begin{array}{l}\text { The polynomial order and } \\
\text { window size for } \\
\text { polynomial fit affects the } \\
\text { result }\end{array}$ & $\begin{array}{l}\text { The polynomial function should } \\
\text { have an order similar to the } \\
\text { spectral data (e.g., } 2^{\text {nd }} \text { order } \\
\text { polynomial function for IR data) } \\
\text { and the window size should be an } \\
\text { odd number and not too small } \\
\text { (keeping the noise) or too large } \\
\text { (changing the spectral shape) }\end{array}$ \\
\hline $\begin{array}{l}\text { Multiplicative scatter } \\
\text { correction (MSC) }\end{array}$ & $\begin{array}{c}\text { Light scattering (Mie } \\
\text { scattering), different } \\
\text { pressure over the sample } \\
\text { when using ATR or probe, } \\
\text { different lengths of optical } \\
\text { path }\end{array}$ & $\begin{array}{l}\text { ATR-FTIR, FTIR, } \\
\text { NIR, Raman, NMR, } \\
\text { UV-Vis }\end{array}$ & $\begin{array}{l}\text { Corrects light scattering } \\
\text { maintaining the same } \\
\text { spectral shape and signal } \\
\text { scale }\end{array}$ & $\begin{array}{c}\text { Need of a reference } \\
\text { spectrum representative of } \\
\text { all measurements }\end{array}$ & $\begin{array}{l}\text { The reference spectrum is } \\
\text { regularly set as the average } \\
\text { spectrum across all training } \\
\text { samples }\end{array}$ \\
\hline $\begin{array}{l}\text { Standard normal variate } \\
\qquad(\mathrm{SNV})^{197}\end{array}$ & $\begin{array}{c}\text { Light scattering (Mie } \\
\text { scattering), different } \\
\text { pressure over the sample } \\
\text { when using ATR or probe, } \\
\text { different lengths of optical } \\
\text { path }\end{array}$ & $\begin{array}{l}\text { ATR-FTIR, FTIR, } \\
\text { NIR, Raman, NMR, } \\
\text { UV-Vis }\end{array}$ & $\begin{array}{l}\text { Corrects light scattering } \\
\text { maintaining the same } \\
\text { spectral shape }\end{array}$ & $\begin{array}{l}\text { Creates negative signals } \\
\text { since the data are } \\
\text { centralized to zero (y- } \\
\text { scale) }\end{array}$ & -- \\
\hline $\begin{array}{c}\text { Spectral } \\
\text { differentiation }\end{array}$ & $\begin{array}{c}\text { Light scattering (Mie } \\
\text { scattering), different } \\
\text { pressure over the sample } \\
\text { when using ATR or probe, } \\
\text { different lengths of optical } \\
\text { path, background } \\
\text { absorption interfering }\end{array}$ & $\begin{array}{l}\text { ATR-FTIR, FTIR, } \\
\text { NIR, Raman, NMR, } \\
\text { UV-Vis }\end{array}$ & $\begin{array}{c}\text { Corrects light scattering } \\
\text { and baseline problems; } \\
\text { highlights smaller spectral } \\
\text { differences }\end{array}$ & $\begin{array}{c}\text { Changes the signal scale, } \\
\text { shifts the data and } \\
\text { increases noise }\end{array}$ & $\begin{array}{l}\text { The order of the derivative } \\
\text { function should be used carefully } \\
\text { to avoid increased noise (usually } \\
1^{\text {st }} \text { or } 2^{\text {nd }} \text { order differentiation is } \\
\text { preferred). The differentiation can } \\
\text { be coupled to Savitzky-Golay } \\
\text { smoothing }\end{array}$ \\
\hline Baseline correction $^{198}$ & $\begin{array}{l}\text { Background absorption } \\
\text { interfering }\end{array}$ & $\begin{array}{l}\text { ATR-FTIR, FTIR, } \\
\text { NIR, Raman, NMR, } \\
\text { UV-Vis, MS }\end{array}$ & $\begin{array}{l}\text { Corrects the baseline } \\
\text { maintaining the same } \\
\text { spectral shape }\end{array}$ & -- & $\begin{array}{l}\text { There are many methods for } \\
\text { baseline correction (e.g., rubber } \\
\text { band, automatic weighted least } \\
\text { squares, Whittaker filter). The } \\
\text { method chosen should be } \\
\text { maintained consistent for all } \\
\text { systems used }\end{array}$ \\
\hline
\end{tabular}


Normalization $^{95}$
Different sample thickness

and concentration
ATR-FTIR, FTIR,

Raman
Avoids influence of non-

desired signals among the
The normalization might

hide signal differences

between samples at

important bands, such as

Amide I and Amide II; and

also may introduce non- 
Figure 1 shows the effect of a pre-processing approach employed for a blood plasma dataset acquired under different experimental conditions (i.e., different systems and operators). In this Figure, the reduction of the spectral differences between the systems is evident after data pre-processing (Savitzky-Golay smoothing, MSC, baseline correction and normalization).

After pre-processing (Table 3), a scaling step should be done, because most classification methods require all the variables (e.g., wavenumbers) in the dataset to be at the same scale in order to work properly.

For spectral data, mean-centring (also referred as "standardization" by Hastie et al. ${ }^{199}$ ) is a very reasonable approach, after which all variables in the dataset will have zero mean. When data contain values represented by different scales (e.g., after data fusion using both IR and Raman spectra), block-scaling should be used, where each block of data (i.e., data from each instrumental technique) would have the same sum-of-squares (normally after meancentring).

Another important aspect of pre-processing is the order in which each step is applied. Pre-processing should be employed in a logical order so that the next pre-processing step is not affected by the previous one. For example, pure spectral differentiation cannot be employed before smoothing, since the spectral differentiation will increase the original noise. Therefore, smoothing should be applied before differentiation. Albeit, Savitzky-Golay routine incorporates smoothing and spectral differentiation so, in practical terms, these can be performed together. To summarise, the suggested order of pre-processing is as follows:

\section{Spectral Truncation}

2. Smoothing

3. Light scattering correction

4. Baseline correction 


\section{Normalization}

6. Scaling

Further details about these pre-processing steps are provided in "Procedure: Data preprocessing" section. When using different instruments but same type of sample, the preprocessing steps should be the same for the data acquired under different circumstances.

\section{Experimental design: data analysis}

Sample splitting. Sample splitting is fundamental for constructing a predictive chemometric model. It consists of a data analysis step performed before construction of a chemometric model, in which a portion of the samples are assigned to a training set, while the remaining samples are assigned to a validation and/or test set. The training set is used for model construction, the validation set for model optimization, and the test set for final model evaluation. The process of dividing the samples in three sets can be performed manually or by computer-based methodologies. Manual splitting can generate biased results, therefore we recommend a computational-based split instead. Some examples of these include random selection, leverage ${ }^{149}$ or the KS algorithm ${ }^{174}$. KS works based on Euclidian distance calculation by firstly assigning the sample with the maximum distance to all other samples to the calibration set, and then by selecting the samples which are as far away as possible from the selected samples to this set, until the designed number of selected samples is reached. This ensures that the calibration model will contain samples that uniformly cover the complete sample space, where no or minimal extrapolation of the remaining samples are necessary; avoiding problems of manual or random selection, such as non-reproducibility and nonrepresentative selection. Usually, the dataset is split with $70 \%$ of the samples assigned for training, $15 \%$ for validation and $15 \%$ for test. In this case, the test set is dependent on the initial group of samples measured, and it is not a regular independent test set where a new set of similar samples are measured. 
256 Exploratory analysis. Exploratory analysis is an important tool to provide an initial 257 assessment of the data. Using exploratory analysis, the analyst can see the clustering patterns and then draw conclusions related to the nature of samples, outliers and experimental errors.

259 One of the most common techniques for exploratory analysis is principal component analysis 260 (PCA), in which the original data are decomposed into a few principal components (PCs) responsible for most of the variance within the original dataset. The PCs are orthogonal to each other and are generated in a decreasing order of explained variance, so that the first PC represents most of the original data variance, followed by the second PC and so on ${ }^{200}$. Mathematically the decomposition takes the form:

$\mathbf{X}=\mathbf{T} \mathbf{P}^{\mathrm{T}}+\mathbf{E}$

where $\mathbf{X}$ represents the pre-processed data (e.g., pre-processed samples' spectra); $\mathbf{T}$ are the scores; $\mathbf{P}$ are the loadings; and $\mathbf{E}$ are the residuals.

The PCA scores represent the variance in the sample direction and they are used to assess similarities/dissimilarities among the samples, thus detecting clustering patterns. The PCA loadings represent the variance in the variable (e.g., wavenumber) direction and they are used to detect which variables show the highest importance for the pattern observed on the scores. The PCA loadings are commonly employed as a tool for searching spectral markers that distinguish different biological classes ${ }^{201}$. The PCA residuals represent the difference between the decomposed and original data and can be used to identify experimental errors. Ideally, the PCA residuals should be random and close to zero, representing a heteroscedastic distribution. Otherwise, they can indicate experimental bias according to a homoscedastic distribution.

For standardization applications, PCA is a fast, intuitive and reliable tool to observe if there are differences between the spectra acquired by different systems. Ideally, if the same 
sample is measured under different conditions (different laboratories, instrument manufacturers or user operators) their PCA scores should be random and completely superposed. If a discrimination pattern is observed on the PCA scores, then it is indicative that the data need standardization. Figure 2 illustrates a PCA scores plot from the same samples (blood plasma of healthy controls) measured using three IR instruments before (Fig. 2a) and after (Fig. 2b) PDS. Even though the samples in Fig. 2a are pre-processed, three different clusters are still evident. After PDS the samples measured using different systems are normalized into a single cluster.

Outlier detection. Outlier detection is important to prevent samples, which differ from the original dataset, from affecting the results using predictive models. Outliers can be attributed to experimental errors, such as inconsistent sample preparation or spectral acquisition, or to larger experimental noise, such as Johnson noise, shot noise, flicker noise and environmental noise. These samples can have large leverage for classification, masking the real signal from the samples of interest; therefore, it is advised that they be removed from the dataset used to train the predictive model. can be utilised among others ${ }^{205}$. One of the most popular and visually intuitive technique for detecting outliers is the Hotelling $\mathrm{T}^{2} v s \mathrm{Q}$ residual test ${ }^{206}$. In this test, a chart is created using the Hotelling $\mathrm{T}^{2}$ values in $x$-axis and the $\mathrm{Q}$ residuals in the $y$-axis, generating a scatter plot. The Hotelling $\mathrm{T}^{2}$ represents the sum of the normalized squared scores, which is the distance from the multivariate mean to the projection of the sample onto the $\mathrm{PCs}^{207}$. The Q residuals represent the sum of squares of each sample in the error matrix, thus measuring the residues between a sample and its projection onto the $\mathrm{PCs}^{207}$. All samples far from the origin of this graph are considered outliers and should be removed one at a time, as the PCA is highly influenced by the samples that are included in the model. Samples with high values in both Hotelling $\mathrm{T}^{2}$ and 
Q residuals are the worst outliers; while samples with high values in only one of these axis are the second worst outliers. Supplementary Method 1 illustrates an example for outlier detection. detection. For example, if the confidence limits is set at a $95 \%$ level, certain amount of datapoints $(5 \%)$ should be statistically outside these boundaries.

Classification. Classification techniques are employed for sample discrimination. Using chemometric analysis, one can distinguish classes of samples based on their spectral features and then make further predictions based on these. The prediction capability of a classification model should be evaluated with external samples (unknown samples) through the calculation of figures of merit, including accuracy (proportion of samples correctly classified considering true positives and true negatives), sensitivity (proportion of positives that are correctly identified) and specificity (proportion of negatives that are correctly identified) ${ }^{208}$. the main classification techniques employed for bio-spectroscopy applications, along with their advantages and disadvantages.

Table 4. Classification techniques.

\begin{tabular}{|c|c|c|}
\hline Classification Technique & Advantage & Disadvantage \\
\hline $\begin{array}{l}\text { Linear discriminant } \\
\text { analysis }(\mathrm{LDA})^{209}\end{array}$ & Simplicity, fast calculation & $\begin{array}{l}\text { Needs data reduction, does not account } \\
\text { for classes having different variance } \\
\text { structures, greatly affected by classes } \\
\text { having different sizes }\end{array}$ \\
\hline $\begin{array}{l}\text { Quadratic discriminant } \\
\text { analysis (QDA) }{ }^{209}\end{array}$ & $\begin{array}{l}\text { Fast calculation, accounts for } \\
\text { classes having different variance } \\
\text { structures, not much affected by } \\
\text { classes having different sizes }\end{array}$ & $\begin{array}{c}\text { Needs data reduction, higher risk of } \\
\text { overfitting }\end{array}$ \\
\hline $\begin{array}{l}\text { Partial least squares } \\
\text { discriminant analysis } \\
\quad(\text { PLS-DA })^{210}\end{array}$ & Fast calculation, high accuracy & $\begin{array}{c}\text { Greatly affected by classes having } \\
\text { different sizes, needs optimization of the } \\
\text { number of latent variables (LVs) }\end{array}$ \\
\hline $\begin{array}{l}\text { K-Nearest Neighbours } \\
\qquad(\mathrm{KNN})^{211}\end{array}$ & $\begin{array}{l}\text { Simplicity, non-parametric, suitable } \\
\text { for large datasets }\end{array}$ & $\begin{array}{c}\text { Time consuming, needs optimization of } \\
\text { the distance calculation method and } k \\
\text { value, highly sensitive to the "curse of } \\
\text { dimensionality"199 }\end{array}$ \\
\hline $\begin{array}{l}\text { Support vector machines } \\
\qquad(\mathrm{SVM})^{212}\end{array}$ & $\begin{array}{l}\text { Non-linear classification nature, } \\
\text { high accuracy }\end{array}$ & $\begin{array}{l}\text { High complexity, high risk of overfitting, } \\
\text { needs optimization of kernel function } \\
\text { and SVM parameters, time consuming }\end{array}$ \\
\hline
\end{tabular}


Artificial neural networks $(\mathrm{ANN})^{213}$

Random forests ${ }^{214}$

Deep learning approaches $^{215}$
Non-linear classification nature, ability to work with incomplete knowledge, high accuracy

Non-linear classification nature, high accuracy, relatively low computational cost

Non-linear classification nature, native feature extraction (e.g., in convolutional neural networks $(\mathrm{CNN}))$, local spatial coherence (CNN), high accuracy
High computational cost, needs optimization of the number of neurons and layers, no interpretability ("black box" model)

High risk of overfitting, needs optimization of the number of trees, no interpretability ("black box" model)

High computational cost, needs hyperparameter optimization, needs large datasets, time consuming, no interpretability ("black box" model)
321

When employing classification techniques, one must follow a parsimony $\operatorname{order}^{216}$, where the simplest algorithms should be used first, reducing the need for more complex algorithms which would require more optimization steps. An order for using these classification algorithms is: $\mathrm{LDA}>\mathrm{PLS}-\mathrm{DA}>\mathrm{QDA}>\mathrm{KNN}>\mathrm{SVM}>\mathrm{ANN}>$ Random forests $>$ Deep learning approaches, from the simplest to the most complex.

$$
\text { techniques in order to reduce data collinearity/redundancy, thus reducing the risk of overfitting }
$$
in the classifier training, and speeding up such training, as there are less variables involved. An additional benefit of such a feature extraction/selection step is to provide spectral markers identification as a "side-effect" (depending on the feature extraction/selection method applied). For feature extraction, the most popular technique is PCA. In this case, a PCA is firstly applied to the data, and then the PCA scores are used as the input variables (instead of the wavenumbers data points) for the classification techniques mentioned above ${ }^{217}$. PLS-DA is also a feature extraction technique ${ }^{210}$, and normally it performs better than a PCA followed by LDA, as the scores from a PCA does not necessarily describe the difference between the samples, but rather the variance in the data. In PLS-DA, a partial least squares (PLS) model is applied to the data in an interactive process reducing the original variables to a few number of LVs, where a LDA is used for classifying the groups ${ }^{218}$. Other discriminant classifiers, in particular QDA, also could be used in this classification step to circumvent problems observed with LDA. For feature 
selection, there are many techniques commonly employed in biological datasets, including genetic algorithm $(\mathrm{GA})^{219}$ and successive projections algorithm (SPA) ${ }^{220}$. The variables (e.g., wavenumbers) selected by these techniques are used as input variables for the classification models described in Table 2. An important advantage of GA is its relatively low-computational cost compared to SPA and reduction of data collinearity. Furthermore, GA-based techniques are intuitive and simple to understand in the algorithmic sense but they also have a nondeterministic nature and require optimization of many parameters. SPA's advantage relies on its deterministic nature, minor parameter optimization and reduction of data collinearity, however, it is very time consuming. For hyperspectral imaging, feature selection can also be performed by Minimum Redundancy Maximum Relevance (mRMR) algorithm ${ }^{221}$, where the selection process is based on maximizing the relevance of extracted features and simultaneously minimize redundancy between them.

Standardization. Data standardization should be employed when a primary classification model is built and new data comes to be predicted from a secondary system (different laboratory or instrument manufacturers), or when there is a change in instrument components (e.g., laser, gratings, etc.) or when the data of the chemometric model are acquired under different circumstances (different analysts, days, instrumental settings, etc.). As previously mentioned, the most common and reliable methods for data standardization are the DS and PDS algorithms. These methods can be found in a few software packages (described in Table 3).

Figure 3 summarises the standardization protocol using DS applied to spectra acquired under different conditions. The first step consists of applying KS algorithm for selecting the number of transfer samples from the primary system as well as the number of training samples for the secondary systems, which is ideally $70 \%$ of the dataset. Thereafter, the DS transform generation algorithm is employed to estimate the transform matrix. The validation set of the 
secondary system is then used with the classification model of the primary system to evaluate the optimum number of transfer samples. This optimization step is repeated depending on the number of transfer samples from the primary system. After this number is defined, the validation set of the secondary system is finally standardized and the final classification model is subsequently applied. This procedure is realized with a certain number of samples measured in all instruments being standardized. This procedure should be realized in as similar manner as possible to reduce spectral differences. After the model is standardized and proper validated, new external samples can be measured in any of the instruments and predicted by the standardized classification model.

For PDS, an extra step is added after defining the number of transfer samples to estimate the optimum window size. The dashed region in Fig. 3 is repeated according to the window size.

For multi-laboratory studies the flowchart depicted in Fig. 4 illustrates how the standardization protocol should be employed.

In Fig. 4, spectra acquired under different experimental conditions are used for a global standardization model. A primary system should be designated and then all spectra from secondary systems are equally pre-processed, followed by an exploratory analysis to assess samples' similarities/dissimilarities, outlier detection, standardization by the method outlined in Figure 3; the final model construction follows last. With this, all sources of variations present in different systems can be included into a general chemometric model. 
- Biological samples (tissue, cells, biofluids)(see Reagent Setup).

A CRITICAL Human samples should be collected with appropriate local institutional review board for ethical approval and adhere to the Declaration of Helsinki principles. Similarly, for studies involving animals, all experiments should be performed in accordance with relevant guidelines and regulations. Ethical approval has to be obtained before any sample collection.

- Optimal cutting temperature (OCT) compound (Agar Scientific, cat. no. AGR1180)

- Liquid nitrogen (BOC, CAS no. 7727-37-9) ! CAUTION Asphyxiation hazard; make sure room is well ventilated. Causes burns; wear face shield, gloves and protective clothing.

- Paraplast Plus paraffin wax (Thermo Fisher Scientific, cat. no. SKU502004)

- Isopentane (Fisher Scientific, cat. no. P/1030/08) ! CAUTION Extremely flammable, 400 irritant, aspiration hazard and toxic; use in a fume hood.

- Distilled water

402

- $\quad$ PBS (10x; MP Biomedicals, cat. no. 0919610)

- Virkon (Antec, DuPont, cat. no. A00960632)

- Trypsin-EDTA (0.05\%, Sigma-Aldrich, Thermo Fisher Scientific cat. no. 25300054)

405

406

\section{Anticoagulants}

407

- EDTA (Thermo Fisher Scientific, BD Vacutainer, cat. no. 02-687-107)

- Sodium citrate (Thermo Fisher Scientific, BD Vacutainer)

- Lithium/sodium heparin (Thermo Fisher Scientific, BD Vacutainer) 
- Formalin, 10\% (vol/vol; Sigma-Aldrich, cat. no. HT501128) ! CAUTION Potential

413 carcinogen, irritant and allergenic; use in a fume hood.

414 - Ethanol (Fisher Scientific, cat. no. E/0600DF/17)

415 - Methanol (Fisher Scientific, cat. no. A456-212) ! CAUTION Toxic vapours; use in a fume 416 hood.

- Acetone (Fisher Scientific, cat. no. A19-1) ! CAUTION Acetone vapors may cause 418 dizziness; use in a fume hood.

- $\quad$ ThinPrep (PreservCyt Solution, Cytyc Corp)

- SurePath (Becton Dickinson Diagnostics)

\section{Dewaxing agents}

423

- Xylene (Sigma-Aldrich, cat. no. 534056) ! CAUTION Potential carcinogen, irritant and 424 allergenic; use in a fume hood.

- Histo-Clear (Fisher Scientific, cat. no. HIS-010-010S) ! CAUTION It is an irritant.

- Hexane (Fisher Scientific, cat. no. 10764371) ! CAUTION Extremely flammable liquid, 427 can cause skin irritation; use protective equipment as required; use in a fume hood.

- Microtome (Thermo Fisher Scientific, cat. no. 902100A; or cat. no. 956651)

431 - Wax dispenser (Electrothermal, cat. no. MH8523B)

- Sectioning bath (Electrothermal, cat. no. MH8517)

- Centrifuge (Thermo Fisher Scientific, cat. no. 75002410)

434 - Desiccator (Thermo Fisher Scientific, cat. no. 5311-0250)

- Desiccant (Sigma-Aldrich, cat. no. 13767)

- Laser power meter (Coherent, cat. no. 1098293) 
- Computer system

439

$440 \quad$ Substrates

A CRITICAL Substrate should be carefully chosen depending on the spectrochemical approach and the experimental mode that will be used. For more details about the choice of substrate see ref ${ }^{4,99}$.

- Low-E slides (Kevley Technologies, CFR)

- $\mathrm{BaF}_{2}$ slides (Photox Optical Systems)

- $\mathrm{CaF}_{2}$ slides (Crystran, cat. no. CAFP10-10-1)

447 - Silicon multi-well plate (Bruker Optics)

- Glass slides (Fisher Scientific, cat. no. 12657956)

- Quartz slides (UQG Optics, cat. no. FQM-2521)

- Aluminum-coated slides (EMF, cat. no. AL134)

451

- Mirrored stainless steel (Renishaw, cat. no. A-9859-1825-01)

452

453 REAGENT SETUP

454 Tissue For FFPE tissue, the excised specimen is immersed in fixative (e.g., formalin), 455 dehydrated in ethanol, cleared in xylene and embedded in paraffin wax. Specimens can then be stored indefinitely at room temperature. For snap-frozen tissue, the specimen is immersed in OCT, followed by cooling of isopentane with liquid $\mathrm{N}_{2}$.

A CRITICAL Snap-frozen tissue should be thawed before analysis. Spectroscopic analysis should be performed directly after excision in case of fresh tissue to avoid sample degradation. 
461

462

463

464

465

466

467

468

469

470

471

472

473

474

475

476

477

478

479

480

481

482

A CRITICAL In case cells are fixed or stored in a preservative solution, a number of washing steps using centrifugation should be followed prior to spectroscopic analysis to remove unwanted signature. If cells are studied alive, optimum living conditions (e.g., growth medium, temperature and $\mathrm{pH}$ ) should be maintained; washing of live cells from medium is also necessary.

Biofluids Biofluids can be collected in designated, sterile tubes using standard operating procedures to achieve uniformity of performance. Preparation of biofluids depends on the sample type and the experiment's objective. If cellular material is not directly studied, it should be removed from the biofluid before storage. Biofluids can be analysed right after their collection or stored at a $-80^{\circ} \mathrm{C}$ freezer.

A CRITICAL If biofluids have been stored in a freezer, it is essential that they are fully thawed before acquiring aliquots for spectroscopic analysis.

A CRITICAL Users are advised to store biofluids in smaller, single-use aliquots at $-80^{\circ} \mathrm{C}$ to avoid repeated freeze-thaw cycles.

\section{EQUIPMENT SETUP}

The user can choose from a range of different instrumental setups and spectral acquisition modes. General information about FTIR systems is provided below. For more details about equipment setup see refs. ${ }^{4,98,99}$.

The FTIR spectrometer can be left on for long periods of time. Before spectral acquisition, the user should check the interferogram signal for amplitude and position and keep a record of the measurements. 
483

484

485

486

487

488

489

490

491

492

493

494

495

496

497

498

499

500

501

502

503

504

A CRITICAL For detectors that require a prior cooling step using liquid nitrogen (e.g., mercury cadmium telluride (MCT) detectors), the signal should be allowed to stabilize for approximately $10 \mathrm{~min}$ before data collection.

A CRITICAL In case that the interferogram signal deviates from the last measurement, realignment or part replacement may be required.

Software: Software for spectral acquisition is typically provided by the manufacturer. Software packages for spectral analysis and data standardization are provided in Table 3.

\section{PROCEDURE}

Sample preparation

1| Prepare the biological samples for spectrochemical analysis using the following steps: option A for FFPE tissue samples, option B for snap-frozen or fresh tissue samples, option C for cells and option D for biofluids.

A CRITICAL Sample preparation is briefly presented in this protocol. More details about sample preparation can be found in refs. ${ }^{4,98,99}$.

\section{(A) Tissue (FFPE) • TIMING 1-1.5 h}

(i) Obtain FFPE tissue blocks.

(ii) Section the whole tissue block using a microtome to obtain tissue sections at desired thickness $(2-10 \mu \mathrm{m})$.

A CRITICAL STEP Cooling of the tissue on an ice block for $10 \mathrm{~min}$ prior to sectioning, hardens the wax and allows easier cutting.

(iii) Float the tissue ribbons in a warm $\mathrm{H}_{2} \mathrm{O}$ bath $\left(40-44^{\circ} \mathrm{C}\right)$ and then deposit onto the substrate of choice. 
(iv) Allow the tissue sections to dry either at room temperature (30 min) or in a $60^{\circ} \mathrm{C}$

506

507

508

509

510

511

512

513

514

515

516

517

518

519

520

521

522

523

524

525

526 oven (10 min).

A CRITICAL STEP The tissue slide may be dried in the oven for longer periods of time, depending on the type of tissue, to ensure optimal, initial melting of the wax.

(v) Dewax the samples by performing three sequential immersions in a dewaxing reagent such as fresh xylene, Histo-Clear solution or hexane (each immersion should last at least $5 \mathrm{~min})$.

$\triangle$ CRITICAL STEP Thorough dewaxing is important for eliminating all spectral peaks attributed to paraffin.

(vi) Immerse the tissue slide in acetone or ethanol ( $5 \mathrm{~min}$ ) to remove the xylene and then left to air-dry.

- PAUSE POINT Slides can be stored in a desiccator at room temperature for at least 1 year.

(B) Tissue (Snap-frozen or fresh) • TIMING $2 \mathrm{~h}+$ drying time ( $3 \mathrm{~h}$ for FTIR only)

$\Delta$ CRITICAL Snap-frozen tissue can be stored at $-80^{\circ} \mathrm{C}$ for several months.

A CRITICAL For fresh tissue, proceed to step 1B(ii).

(i) Acquire snap-frozen tissue from freezer and place onto a cryostat (30 min) to allow the tissue to reach the cryostat's temperature $\left(-20^{\circ} \mathrm{C}\right)$.

(ii) Use a cryostat to obtain tissue sections at desired thickness $(8-10 \mu \mathrm{m})$.

(iii) Deposit the tissue sections onto an appropriate substrate before spectra are collected (see a list of substrates in the Materials-Equipment section).

A CRITICAL For FTIR studies the tissue sections need to dry for at least $3 \mathrm{~h}$ to remove the $\mathrm{H}_{2} \mathrm{O}$ interference from the IR spectra. 
A CRITICAL Exposure to light should be minimised to prevent sample degradation due to oxidation.

\section{(C) Cells (fixed or live) $\bullet$ TIMING $30 \mathrm{~min}+$ desiccation time ( $3 \mathrm{~h}$ for FTIR only)}

A CRITICAL If you are working with fixed cells, do step 1C(i) and then proceed to step $1 \mathrm{C}(\mathrm{iii})$. If you are working with live cells, proceed to step $1 \mathrm{C}(\mathrm{ii})$

(i) Wash fixed cells to remove the fixative or preservative solution as these chemicals cause spectral interference in the fingerprint region. Three sequential washes with distilled $\mathrm{H}_{2} \mathrm{O}$ or PBS have been shown to remove unwanted peaks.

(ii) Detach cultured cells from the growth substrate adding 2-3 $\mathrm{mL}$ of fresh warm trypsin/EDTA solution to the side wall of the flask; gently swirl the contents to cover the cell layer. Wash with warmed sterile PBS to remove the medium and trypsin $(\times 3$ times; gentle centrifuge at $300 \mathrm{~g}$ for $7 \mathrm{~min})$.

$\triangle$ CRITICAL STEP All reagents should be warmed to $37^{\circ} \mathrm{C}$ to reduce the shock to cells and maintain morphology.

(iii) After the final wash, resuspend the remaining cell pellet in distilled $\mathrm{H}_{2} \mathrm{O}(\sim 50-100$ $\mu \mathrm{L}$ ) and mount onto a substrate of choice; allow sample to dry before analysis.

$\triangle$ CRITICAL STEP The final suspension of cells $(\sim 50-100 \mu \mathrm{L})$ should be evenly deposited on the slide either by cytospinning or by micro-pipetting. For cytospinning, take a maximum volume of $200 \mu \mathrm{L}$ of cells in suspension (spin-fixed cells at $800 \mathrm{~g}$ for $5 \mathrm{~min}$ ). After spinning, leave the slide to air-dry.

A CRITICAL For FTIR studies the sample needs to dry for at least $3 \mathrm{~h}$. 
$549 \Delta$ CRITICAL If biofluids are analysed fresh, immediately after collection, continue to step 1D(ii).

(i) Acquire biofluids from the $-80^{\circ} \mathrm{C}$ freezer and allow them to fully thaw.

(ii) Mix or gently vortex the sample before obtaining the desired volume for analysis.

553

554

(iii) Deposit the biological fluid onto an appropriate substrate.

$\triangle$ CRITICAL STEP For ATR-FTIR spectroscopic studies, an alternative option is to deposit the sample directly on the ATR crystal instead of a substrate if the instrumentation setting allows (i.e., if crystal is facing upwards). However, if the sample is sufficiently thick $(>2-3 \mu \mathrm{m})$ to avoid substrate interference, then the use of a holding substrate is advantageous as it allows measurements from multiple locations as well as longer storage.

$\triangle$ CRITICAL STEP For FTIR studies the sample needs to dry adequately before spectroscopic analysis (50 $\mu \mathrm{L}$ dry within approximately $1 \mathrm{~h}$ at room temperature). Drying can be sped up by using a gentle stream of air over the sample at a specific flow rate (in a sterile laminal flow hood). 
$571 \Delta$ CRITICAL Spectrochemical information can be collected as follows for FTIR spectroscopy.

$\triangle$ CRITICAL Spectral acquisition is briefly presented in this protocol. More details can be 574 found in refs. ${ }^{4,98,99}$.

2| Optimise the settings before each new study to increase the SNR (see 'Experimental design: spectral acquisition').

$\triangle$ CRITICAL STEP Some of the parameters that need to be adjusted include the resolution, spectral range, co-additions, aperture size, interferometer mirror velocity, and interferogram zero-filling.

$\triangle$ CRITICAL STEP To improve reproducibility and decrease differences between the data collected by different operators, the spectral resolution should be set constant, since it can cause major differences between data collected across different experimental setups.

$\triangle$ CRITICAL STEP The pressure applied on the sample in the ATR mode affects the signal intensity (i.e., absorbance) between data collected by different instruments and operators. Thus, the pressure applied on the sample should be as similar as possible across different experimental setups to reduce differences between the spectra collected. Depending on the sampling mode that has been chosen (ATR-FTIR, transmission or transflection), deposit the sample onto the appropriate holding substrate.

3 | Acquire a background spectrum to account for atmospheric changes.

$\triangle$ CRITICAL STEP This should be done before every sample. 
4 | Load the sample and visualise the region of interest; information can then be acquired either as point map or as image maps.

A CRITICAL Typically, 5-25 point spectra are collected per sample while for image maps the step size should be the same or smaller than the selected aperture size divided by two. Sampling can be performed with 6 replicates in 3 levels.

\section{Data quality evaluation $\bullet$ TIMING $15 \mathrm{~min}-4 \mathrm{~h}$ (depending on the size of the dataset)}

5| Evaluate the raw data using quality tests to identify anomalous spectra or biased patterns before applying pre-processing. This can be made by visual inspection of the collected spectra followed by Hotelling $\mathrm{T}^{2}$ versus $\mathrm{Q}$ residuals charts (see Supplementary Method 1) using only the mean-centred data, and analysis of PCA residuals. Samples far from the origin of the Hotelling $\mathrm{T}^{2}$ versus $\mathrm{Q}$ residuals chart should be removed, and PCA residuals should be random and close to zero. Further instructions about data quality evaluation can be found at "Experimental Design: data quality evaluation" section.

\section{Data pre-processing $\bullet$ TIMING $15 \mathrm{~min}-4 \mathrm{~h}$ (depending on the size of the dataset)}

A CRITICAL Steps 6-11 below can be modified depending on the nature of the dataset. Table 1 provides more details about these pre-processing steps. In case of an ATR-FTIR dataset where samples were acquired and analysed under different experimental conditions, the preprocessing method should follow this order:

$6 \mid$ Cutting at biofingerprint region $\left(900-1800 \mathbf{c m}^{-1}\right)$. Truncate the spectra to the biofingerprint region, to eliminate atmospheric interference present in other regions of the spectra. 
7| Savitzky-Golay smoothing for removing spectral-noise. Window size varies according to the size of the spectra dataset (e.g., wavenumber). The window size should be an odd number, since a central data point is required for the smoothing process. Try different window sizes from 3 to 21 and observe how the spectra change (in shape) and how the noise is reduced. Use the smallest window that removes the noise considerably whilst maintaining the original spectral shape. Using a spectral resolution of $4 \mathrm{~cm}^{-1}$, the biofingerprint region $\left(900-1800 \mathrm{~cm}^{-1}\right)$ usually contains 235 wavenumbers. In that case, a window size of 5 points should be used. The polynomial order for Savitzky-Golay fitting should be $2^{\text {nd }}$ order for IR spectroscopy due to the band shape.

8 | Light scattering correction using either multiplicative scatter correction (MSC), SNV or $2^{\text {nd }}$ derivative. First try using MSC or SNV, as MSC maintains the spectral scale and both methods maintain the original spectral shape. If the results are not satisfactory (e.g., classification accuracy $<75 \%)$, try using the $2^{\text {nd }}$ derivative spectra.

9| Perform baseline correction using automatic weighted least squares or rubber band baseline correction. If spectral differentiation is applied as light scattering correction method, baseline correction is not necessary.

10 Normalization Normalize the spectrum to the amide I peak or amide II peak, or perform a vector normalization $(2-$ Norm, length $=1)$ to correct different scales across spectra (e.g., due to different sample thicknesses when using FTIR in transmission mode).

11 Scaling Mean-centre the data for each variable, and divide this value by the variable standard deviation. In case of data fusion, block-scaling should be used. 
Data analysis

\section{Exploratory analysis. $\bullet$ TIMING $1 \mathrm{~h}-4 \mathrm{~d}$ (depending on the data size)}

12 Determine whether a standardisation procedure is necessary by performing PCA. The PCA scores plot (PC1 vs PC2) should generate a unique clustering pattern for the same type of sample. If two or more clusters are observed for the same type of sample measured under different experimental conditions, then a standardisation procedure is necessary (see Figure 2).

\section{Outlier detection. $\bullet$ TIMING $1 \mathrm{~h}-1 \mathrm{~d}$ (depending on the data size)}

13 Apply PCA to the dataset and then estimate the Q residuals and Hotelling $\mathrm{T}^{2}$ values. Use the chart of Q residuals versus Hotelling $\mathrm{T}^{2}$ to identify outliers. The outliers (e.g., cosmic rays, artefacts, low signal spectra and substrate only (non-tissue) spectra) should be removed from the data set before proceeding to the next steps.

\section{Sample split. • TIMING $1-4 \mathrm{~h}$ (depending on the data size)}

14 Separate the samples that will be used for the training and the test sets. Sample split should be performed before construction of standardization of multivariate classification models. The samples can be split into training (70\%) and test (30\%) sets, using a cross-validated model; or split into training (70\%), validation $(15 \%)$ and test (15\%) sets without using cross-validation. To maintain consistency and account for a well-balanced training model, KS algorithm should be employed to separate the samples into each set. KS algorithm is freely available at https://doi.org/10.6084/m9.figshare.7607420.v1.

\section{Standardization. $\bullet$ TIMING $1 \mathrm{~h}-4 \mathrm{~d}$ (depending on the data size)}

A CRITICAL Standardization methods should be employed in the following order: DS > PDS (DS should be done before PDS), since the latter is more complex and 
requires an additional optimization step (window size optimization). The data from the secondary response should be separated into training (70\%), validation (15\%) and test (15\%) sets using KS algorithm. The number of transfer samples should be firstly optimized using the validation set from the secondary response. Then, when employing PDS, the window size should be optimized according to the size of the dataset.

15 Use DS to vary the number of transfer samples from 10-100\% of the training set from the primary system. Use the validation set from the secondary instrument to find the optimum number of transfer samples using the misclassification rate as cost function.

16 Perform PDS using the optimum number of samples found with DS. Test different window sizes using the validation set from the secondary system with the misclassification rate as cost function. The window size should vary from 3-29 for a spectral set with resolution of $4 \mathrm{~cm}^{-1}$ in the biofingerprint region (235 variables).

\section{Model construction. $\bullet$ TIMING $1 \mathrm{~h}-4 \mathrm{~d}$ (depending on the data size)}

A CRITICAL Feature extraction (e.g., by means of PCA) or feature selection (e.g., by means of GA or SPA) should be employed to reduce data collinearity and speed up data processing and analysis time. PLS-DA is already a feature extraction method, thus the performance of prior feature extraction is not necessary in this case. The classification technique employed must follow a parsimony order: LDA>PLSDA $>$ QDA $>$ KNN $>$ SVM $>$ ANN $>$ Random forests $>$ Deep learning approaches.

17 |Apply the feature extraction or selection technique. The optimization of the number of PCs during PCA can be performed using an external validation set (15\% of the original dataset) or using cross-validation (leave-one-out for small dataset [ppl samples] or venetian blinds [sample splitting: 10] for large datasets [>20 samples]). GA should be realized three-times starting from different initial populations and the best result using an external validation set (15\% of the original dataset) should be used. Cross-over 
probability should be set for $40 \%$ and mutation probability should be set for $1-10 \%$ according to the size of the dataset.

18 The classification method should be employed using optimization with an external validation set or cross-validation, especially for selecting the number of latent variables of PLS-DA and the kernel parameters for SVM. The kernel function for SVM should be RBF kernel, due to its adaptation to different data distributions. To avoid overfitting, cross-validation should be always performed during model construction to estimate the best RBF parameters.

\section{? TROUBLESHOOTING}

Spectral acquisition: Spectral resolution, spectral range, SNR and signal aperture should be optimized during experimental setup. Operators using different systems should try to keep these parameters constant to reduce spectral differences.

Data pre-processing: To reduce spectral differences, the same data pre-processing should be applied for spectra acquired in different systems.

Standardization: To improve the prediction capability of the classification model, the primary system used should be the one with highest spectral resolution and smallest noise, since all data from the secondary systems will be standardized to this pattern.

\section{- TIMING}

\section{Sample preparation:}

Step 1(A) Tissue (FFPE): 1-1.5 h

1(B) Tissue (Snap-frozen or fresh): $2 \mathrm{~h}+$ drying time $(3 \mathrm{~h}$ )

1(C) Cells (fixed or live): $30 \mathrm{~min}+$ desiccation time $(3 \mathrm{~h})$

1(D) Biofluids (frozen or fresh): $5 \mathrm{~min}+$ thawing (20 min) + drying (1-1.5 h) 
712 Steps 2-4, Spectral acquisition: $1 \mathrm{~s}-5 \mathrm{~min}$ per spectrum (depending on the instrument and spectral acquisition configurations)

714 Step 5, Data quality evaluation: 15 min $-4 \mathbf{h}$ (depending on the size of the dataset)

715 Steps 6-11, Data pre-processing: $15 \mathrm{~min}-4 \mathrm{~h}$

716 Data analysis:

717 Step 12, Exploratory analysis: $1 \mathrm{~h}-4 \mathrm{~d}$

718 Step 13, Outlier detection: $1 \mathrm{~h}-1 \mathrm{~d}$

719 Step 14, Sample split: 1- 4h (depending on sample size)

720 Step 15-16, Standardization: $1 \mathrm{~h}-4 \mathrm{~d}$

721 Step 17-18, Model construction: $1 \mathrm{~h}-4 \mathrm{~d}$

722

723

724

725

726

727

728

729

730

Table 4. Experimental conditions for pilot study.

\section{ANTICIPATED RESULTS}

To illustrate how this protocol can be used in practice, we conducted a pilot study to evaluate the effect of different instrument manufacturers and operators towards spectral acquisition of healthy controls and ovarian cancer samples based on blood plasma (5 healthy controls with 10 spectra per sample; 5 ovarian cancers with 10 spectra per sample) for a binary classification model using ATR-FTIR spectroscopy. All specimens were collected with ethical approval obtained at Royal Preston Hospital UK (16/EE/0010). Table 4 summarizes the experimental conditions in which the experiments were performed.

\begin{tabular}{lllllll}
\hline Instrument & Operator & Spectral range & $\begin{array}{l}\text { Number } \\
\text { co-additions }\end{array}$ & $\begin{array}{l}\text { Spectral } \\
\text { resolution }\end{array}$ & $\begin{array}{l}\text { Room } \\
\text { temperature }\end{array}$ & $\begin{array}{l}\text { Air } \\
\text { humidity }\end{array}$ \\
\hline $\mathrm{A}$ & 1 & $4000-400 \mathrm{~cm}^{-1}$ & 32 & $4 \mathrm{~cm}^{-1}$ & $23.0^{\circ} \mathrm{C}$ & $23 \%$ \\
& 2 & $4000-400 \mathrm{~cm}^{-1}$ & 32 & $4 \mathrm{~cm}^{-1}$ & $23.4^{\circ} \mathrm{C}$ & $26 \%$ \\
$\mathrm{~B}$ & 1 & $4000-400 \mathrm{~cm}^{-1}$ & 32 & $4 \mathrm{~cm}^{-1}$ & $24.0^{\circ} \mathrm{C}$ & $26 \%$ \\
& 2 & $4000-400 \mathrm{~cm}^{-1}$ & 32 & $4 \mathrm{~cm}^{-1}$ & $24.9^{\circ} \mathrm{C}$ & $24 \%$ \\
$\mathrm{C}$ & 1 & $4000-400 \mathrm{~cm}^{-1}$ & 48 & $4 \mathrm{~cm}^{-1}$ & $22.5^{\circ} \mathrm{C}$ & $28 \%$ \\
& 2 & $4000-400 \mathrm{~cm}^{-1}$ & 48 & $1 \mathrm{~cm}^{-1}$ & $22.8^{\circ} \mathrm{C}$ & $26 \%$ \\
\hline
\end{tabular}



instrument $\mathrm{C}$ was an ATR-FTIR Thermo Scientific Nicolet iS10. The spectra were collected for the same types of samples within three different days (operator 1: instrument A in day 1, instrument $\mathrm{B}$ in day 3 , and instrument $\mathrm{C}$ in day 2; operator 2: instrument $\mathrm{A}$ in day 2, instrument

$\mathrm{B}$ in day 1 , and instrument $\mathrm{C}$ in day 3 ) and across two different laboratories (instrument $\mathrm{A}$ and

$\mathrm{B}$ in laboratory 1 and instrument $\mathrm{C}$ in laboratory 2). Each operator prepared the samples individually from the same bulk, and measured them individually. Spectral acquisition times were around $30 \mathrm{~s}$ for instruments $\mathrm{A}$ and $\mathrm{B}$, and $40 \mathrm{~s}$ for instrument $\mathrm{C}$.

\section{Effect of different instruments}

Three different ATR-FTIR spectrometers were used to analyse the samples. Data were pre-processed by truncating at the biological fingerprint region $\left(900-1800 \mathrm{~cm}^{-1}\right)$, followed by Savitzky-Golay smoothing (window of 15 points, $2^{\text {nd }}$ order polynomial function), MSC, baseline correction using automatic weighted least squares and vector normalization (2-Norm, length $=1)$. Each data set $(\mathrm{A}, \mathrm{B}$ and $\mathrm{C})$ was pre-processed individually. The raw and preprocessed spectra for healthy controls and ovarian cancer samples are depicted in Supplementary Figure 1. All spectra collected by the three instrument maintained the same spectral shape, indicating that the chemical information stayed the same; however, large differences between the absorbance intensity were observed between instrument $\mathrm{C}$ and the others (A, B), being caused due to different pressures applied on the sample in the ATR module. The pressure applied to keep the sample in contact with the ATR crystal directly affects the spectral signal intensity, which for instrument A and B (same manufactures) were somewhere controlled by a contra weight, while for instrument $\mathrm{C}$ the pressure was set based on a mechanical screw on the device, thus being biased by the operator usage. The absorbance intensity variation between A and B is observed for this same reason, but in a minor scale. 
756 Outlier detection was performed using a Hotelling $\mathrm{T}^{2}$ versus Q residual test (Supplementary 757 Figure 2).

(i) Classification. Classification was performed using PCA-LDA (10 PCs, explained variance of 99.21\%). Fig. 5a depicts the discriminant function (DF) score plot for PCA-LDA using only the primary system (ATR-FTIR A). As observed, there is an almost perfect separation between the samples from the two classes (accuracy $=100 \%$, sensitivity $=100 \%$, specificity $=100 \%$ ). However, when the spectra acquired using instruments $\mathrm{B}$ and $\mathrm{C}$ are predicted using the model for $\mathrm{A}$, the results decreased significantly (accuracy $=66.7 \%$, sensitivity $=83.2 \%$, specificity $=48.9 \%)($ Fig. $5 b)$, necessitating the use of a standardization procedure.

(ii) Standardization. Standardization was employed using both DS and PDS in order to compare the two methods. The number of transfer samples for DS was optimized according to the misclassification rate obtained for the validation set using the secondary system (Fig. 6a). An optimum number corresponding to $80 \%$ of the samples in the training set of the primary system (55 transfer samples) was obtained, resulting to a misclassification rate of $22.2 \%$ in the validation set of the secondary system. This improved the accuracy (77.8\%) and specificity (80.0\%). Sensitivity decreased to $75.0 \%$, which is an acceptable value. The results after DS are better balanced than without standardization. Fig. $6 \mathrm{~b}$ shows the DF plot for the PCA-LDA model using the training of the primary system and prediction with the secondary system after DS.

PDS was also applied. The number of transfer samples was maintained as 55 (80\% of the primary training set) and the window size was optimized by using the validation set of the secondary system. An optimum window size of 23 wavenumbers was selected with a misclassification rate of $25.9 \%$ (Fig. 6c). The accuracy, sensitivity and specificity using PDS 
were $74.1 \%, 71.4 \%$ and $75.0 \%$, respectively. The DS presented a slightly higher performance than PDS for this dataset. However, DS generated some outliers not observed before, while PDS did not. Thus, in general, PDS provided a better standardization of the data. The PCALDA DF plot after PDS is depicted in Fig. 6d.

\section{Effect of different operators}

The effect of different user operators acquiring spectra from the same samples using the same instruments was also evaluated. Similarly to before, data were pre-processed by cutting the biological fingerprint region $\left(900-1800 \mathrm{~cm}^{-1}\right)$, followed by Savitzky-Golay smoothing (window of 15 points, $2^{\text {nd }}$ order polynomial function), MSC, baseline correction using automatic weighted least squares and vector normalization $(2$-Norm, length $=1)$. Each dataset was pre-processed individually. All raw and pre-processed spectra varying operators are depicted in Supplementary Figures 4 and 5. Outlier detection was performed using a Hotelling $\mathrm{T}^{2}$ versus $\mathrm{Q}$ residual test (Supplementary Figure 7). The PCA scores plots for the pre-processed spectra are depicted in Supplementary Figure 6. The main difference between the operators was observed for instrument C Supplementary Figure 5, since the spectral resolutions used by them were different, which can cause major data distortion.

(i) Classification. Classification was performed using PCA-LDA (10 PCs, explained variance of 98.62\%). Fig. 7a depicts the DF score plot for PCA-LDA using only the primary system (Operator 1). There is a significant separation between the samples from the two classes $($ accuracy $=88.4 \%$, sensitivity $=77.3 \%$, specificity $=100 \%)$. When the spectra acquired by Operator 2 are predicted using the model for Operator 1, the results decreased (accuracy = $75.6 \%$, sensitivity $=66.7 \%$, specificity $=84.6 \%)($ Fig. $7 \mathrm{~b})$, which again necessitates the use of a standardization procedure. 
(ii) Standardization. DS and PDS were employed as standardization methods. The

805

806

807

808

809

810

811

812

814

815

816

817

number of transfer samples for DS was optimized according to the misclassification rate obtained for the validation set using the secondary system (Operator 2) (Fig. 8a). An optimum number of 59 transfer samples (30\% of the samples in the training set of the primary system [Operator 1]) was obtained, resulting in a misclassification rate of $17.8 \%$ in the validation set of the secondary system. This improved the accuracy (82.2\%), sensitivity $(69.6 \%)$ and specificity (95.5\%) compared to the results without DS. Fig. 8b shows the DF plot for the PCALDA model using the training of the primary system and prediction with the secondary system after DS.

The number of transfer samples was maintained as 59 for PDS; and the window size was optimized by using the validation set of the secondary system. An optimum window size of 23 wavenumbers was selected with a misclassification rate of $22.2 \%$ (Fig. 8c). The accuracy, sensitivity and specificity using PDS were $77.8 \%, 100 \%$ and $54.5 \%$, respectively. Although DS obtained an average better classification performance than PDS for this dataset, it also generated some outliers as mentioned before. For this reason, the results after PDS seem better standardized. The PCA-LDA DF plot after PDS is depicted in Fig. 8d.

\section{Acknowledgements}

CLMM would like to thank Coordenação de Aperfeiçoamento de Pessoal de Nível Superior (CAPES) - Brazil (grant 88881.128982/2016-01) for financial support. This work was supported in part in FLM's laboratory by The Engineering and Physical Sciences Research Council (EPSRC; Grant Nos: EP/K023349/1 and EP/K023373/1). MP would like to acknowledge Rosemere Cancer Foundation for funding. 
Author contributions

828

F.L.M. is the principal investigator who conceived the idea for the manuscript;

829

C.L.M.M. and M.P. wrote the manuscript. All co-authors contributed recommendations and

830 provided feedback and changes to the manuscript; and, C.L.M.M., M.P. and F.L.M. brought

831 together the text and finalized the manuscript.

832 Competing financial interests

833 The authors declare no competing financial interest.

834 Data availability statement

835 The datasets generated during and/or analysed during the current study are available

836 from the corresponding authors on reasonable request.

837 
8391 Baker, M. J. et al. Clinical applications of infrared and Raman spectroscopy: state of play and future challenges. Analyst 143, 1735-1757 (2018).

8412 Melin, A. M., Perromat, A. \& Déléris, G. Pharmacologic application of Fourier transform IR spectroscopy: in vivo toxicity of carbon tetrachloride on rat liver. Biopolymers 57, 160-168 (2000).

3 Eliasson, C. \& Matousek, P. Noninvasive authentication of pharmaceutical products through packaging using spatially offset Raman spectroscopy. Anal Chem 79, 1696-1701 (2007).

4 Baker, M. J. et al. Using Fourier transform IR spectroscopy to analyze biological materials. Nat Prot 9, 1771-1791 (2014).

5 Llabjani, V. et al. Polybrominated diphenyl ether-associated alterations in cell biochemistry as determined by attenuated total reflection Fourier-transform infrared spectroscopy: a comparison with DNA-reactive and/or endocrine-disrupting agents. Environ Sci Technol 43, 3356-3364 (2009).

6 Hofmann-Wellenhof, B., Lichtenegger, H. \& Collins, J. Global positioning system: theory and practice. Springer Science \& Business Media (2012).

$7 \quad$ Morris, P. \& Perkins, A. Diagnostic imaging. Lancet 379, 1525-1533 (2012).

8 Lee, S. S. et al. Crohn disease of the small bowel: comparison of CT enterography, MR enterography, and small-bowel follow-through as diagnostic techniques. Radiology 251, 751761 (2009).

9 Lagleyre, S. et al. Reliability of high-resolution CT scan in diagnosis of otosclerosis. Otol Neurotol 30, 1152-1159 (2009).

10 Kalita, J. \& Misra, U. Comparison of CT scan and MRI findings in the diagnosis of Japanese encephalitis. J Neurol Sci 174, 3-8 (2000).

11 Schrevens, L., Lorent, N., Dooms, C. \& Vansteenkiste, J. The role of PET scan in diagnosis, staging, and management of non-small cell lung cancer. Oncologist 9, 633-643 (2004).

12 Jagust, W., Reed, B., Mungas, D., Ellis, W. \& Decarli, C. What does fluorodeoxyglucose PET imaging add to a clinical diagnosis of dementia? Neurology 69, 871-877 (2007).

13 Zhou, M. et al. Clinical utility of breast-specific gamma imaging for evaluating disease extent in the newly diagnosed breast cancer patient. Am J Surg 197, 159-163 (2009).

14 Wallace, B. A. et al. Biomedical applications of synchrotron radiation circular dichroism spectroscopy: identification of mutant proteins associated with disease and development of a reference database for fold motifs. Faraday Discuss 126, 237-243 (2004).

15 Greenfield, N. J. Using circular dichroism spectra to estimate protein secondary structure. Nat Protoc 1, 2876 (2006).

16 Micsonai, A. et al. Accurate secondary structure prediction and fold recognition for circular dichroism spectroscopy. Proc Natl Acad Sci USA 112, E3095-E3103 (2015).

17 Miles, A. J. \& Wallace, B. A. Circular dichroism spectroscopy of membrane proteins. Chem Soc Rev 45, 4859-4872 (2016).

18 Brown, J. Q., Vishwanath, K., Palmer, G. M. \& Ramanujam, N. Advances in quantitative UVvisible spectroscopy for clinical and pre-clinical application in cancer. Curr Opin Biotechnol 20, 119-131 (2009).

19 Yang, P.-W. et al. Visible-absorption spectroscopy as a biomarker to predict treatment response and prognosis of surgically resected esophageal cancer. Sci Rep 6, 33414 (2016).

20 World Health Organization. Fluorescence microscopy for disease diagnosis and environmental monitoring. (2005).

21 Shahzad, A. et al. Diagnostic application of fluorescence spectroscopy in oncology field: hopes and challenges. Appl Spectrosc Rev 45, 92-99 (2010).

22 Sieroń, A. et al. The role of fluorescence diagnosis in clinical practice. Onco Targets Ther $\mathbf{6}$, 977 (2013). 
23 Shin, D., Vigneswaran, N., Gillenwater, A. \& Richards-Kortum, R. Advances in fluorescence imaging techniques to detect oral cancer and its precursors. Future Oncol 6, 1143-1154 (2010).

24 Shahzad, A. et al. Emerging applications of fluorescence spectroscopy in medical microbiology field. J Transl Med 7, 99 (2009).

25 Möller-Hartmann, W. et al. Clinical application of proton magnetic resonance spectroscopy in the diagnosis of intracranial mass lesions. Neuroradiology 44, 371-381 (2002).

26 Gowda, G. N. et al. Metabolomics-based methods for early disease diagnostics. Expert Rev Mol Diagn 8, 617-633 (2008).

27 Frisoni, G. B., Fox, N. C., Jack, C. R., Scheltens, P. \& Thompson, P. M. The clinical use of structural MRI in Alzheimer disease. Nature reviews. Neurology 6, 67-77 (2010).

28 Chan, A. W. et al. $1 \mathrm{H}-\mathrm{NMR}$ urinary metabolomic profiling for diagnosis of gastric cancer. $\mathrm{Br} \mathrm{J}$ Cancer 114, 59 (2016).

29 Palmnas, M. S. \& Vogel, H. J. The future of NMR metabolomics in cancer therapy: towards personalizing treatment and developing targeted drugs? Metabolites 3, 373-396 (2013).

30 Patil, P. \& Dasgupta, B. Role of diagnostic ultrasound in the assessment of musculoskeletal diseases. Ther Adv Musculoskelet Dis 4, 341-355 (2012).

31 Navani, N. et al. Lung cancer diagnosis and staging with endobronchial ultrasound-guided transbronchial needle aspiration compared with conventional approaches: an open-label, pragmatic, randomised controlled trial. Lancet Respir Med 3, 282-289 (2015).

32 Menon, U. et al. Sensitivity and specificity of multimodal and ultrasound screening for ovarian cancer, and stage distribution of detected cancers: results of the prevalence screen of the UK Collaborative Trial of Ovarian Cancer Screening (UKCTOCS). Lancet Oncol 10, 327340 (2009).

33 Smith-Bindman, R. et al. Endovaginal ultrasound to exclude endometrial cancer and other endometrial abnormalities. Jama 280, 1510-1517 (1998).

34 Gajjar, K. et al. Diagnostic segregation of human brain tumours using Fourier-transform infrared and/or Raman spectroscopy coupled with discriminant analysis. Anal Methods $\mathbf{5}$, 89-102 (2013).

35 Bury, D. et al. Phenotyping Metastatic Brain Tumors Applying Spectrochemical Analyses: Segregation of Different Cancer Types. Anal Lett, 1-2 (2018).

36 Hands, J. R. et al. Attenuated Total Reflection Fourier Transform Infrared (ATR-FTIR) spectral discrimination of brain tumour severity from serum samples. J Biophotonics 7, 189-199 (2014).

37 Hands, J. R. et al. Brain tumour differentiation: rapid stratified serum diagnostics via attenuated total reflection Fourier-transform infrared spectroscopy. J Neuro-oncol 127, 463472 (2016).

38 Walsh, M. J., Kajdacsy-Balla, A., Holton, S. E. \& Bhargava, R. Attenuated total reflectance Fourier-transform infrared spectroscopic imaging for breast histopathology. Vib Spectrosc 60, 23-28 (2012).

39 Lane, R. \& Seo, S. S. Attenuated Total Reflectance Fourier Transform Infrared Spectroscopy Method to Differentiate Between Normal and Cancerous Breast Cells. J Nanosci Nanotechnol 12, 7395-7400 (2012).

40 Backhaus, J. et al. Diagnosis of breast cancer with infrared spectroscopy from serum samples. Vib Spectrosc 52, 173-177 (2010).

41 Wang, J.-S. et al. FT-IR spectroscopic analysis of normal and cancerous tissues of esophagus. World J Gastroenterol 9, 1897 (2003).

42 Maziak, D. E. et al. Fourier-transform infrared spectroscopic study of characteristic molecular structure in cancer cells of esophagus: an exploratory study. Cancer Detect Prev 31 (2007). 
43 Mclntosh, L. M. et al. Infrared spectra of basal cell carcinomas are distinct from non-tumorbearing skin components. J Investig Dermatol 112, 951-956 (1999).

44 McIntosh, L. M. et al. Towards non-invasive screening of skin lesions by near-infrared spectroscopy. J Investig Dermatol 116, 175-181 (2001).

45 Mostaço-Guidolin, L. B., Murakami, L. S., Nomizo, A. \& Bachmann, L. Fourier transform infrared spectroscopy of skin cancer cells and tissues. App/ Spectrosc Rev 44, 438-455 (2009).

46 Mordechai, S. et al. Possible common biomarkers from FTIR microspectroscopy of cervical cancer and melanoma. J Microsc 215, 86-91 (2004).

47 Hammody, Z., Sahu, R. K., Mordechai, S., Cagnano, E. \& Argov, S. Characterization of malignant melanoma using vibrational spectroscopy. Sci World J 5, 173-182 (2005).

48 Kondepati, V. R., Keese, M., Mueller, R., Manegold, B. C. \& Backhaus, J. Application of nearinfrared spectroscopy for the diagnosis of colorectal cancer in resected human tissue specimens. Vib Spectrosc 44, 236-242 (2007).

49 Rigas, B., Morgello, S., Goldman, I. S. \& Wong, P. Human colorectal cancers display abnormal Fourier-transform infrared spectra. Proc Natl Acad Sci USA 87, 8140-8144 (1990).

50 Yao, H., Shi, X. \& Zhang, Y. The Use of FTIR-ATR Spectrometry for Evaluation of Surgical Resection Margin in Colorectal Cancer: A Pilot Study of 56 Samples. J Spectrosc 2014, 4 (2014).

51 Lewis, P. D. et al. Evaluation of FTIR Spectroscopy as a diagnostic tool for lung cancer using sputum. BMC Cancer 10, 640 (2010).

52 Akalin, A. et al. Classification of malignant and benign tumors of the lung by infrared spectral histopathology (SHP). Lab Invest 95, 406 (2015).

53 Großerueschkamp, F. et al. Marker-free automated histopathological annotation of lung tumour subtypes by FTIR imaging. Analyst 140, 2114-2120 (2015).

54 Owens, G. L. et al. Vibrational biospectroscopy coupled with multivariate analysis extracts potentially diagnostic features in blood plasma/serum of ovarian cancer patients. $J$ Biophotonics 7, 200-209 (2014).

55 Gajjar, K. et al. Fourier-transform infrared spectroscopy coupled with a classification machine for the analysis of blood plasma or serum: a novel diagnostic approach for ovarian cancer. Analyst 138, 3917-3926 (2013).

56 Theophilou, G., Lima, K. M. G., Martin-Hirsch, P. L., Stringfellow, H. F. \& Martin, F. L. ATRFTIR spectroscopy coupled with chemometric analysis discriminates normal, borderline and malignant ovarian tissue: classifying subtypes of human cancer. Analyst 141, 585-594 (2016).

57 Mehrotra, R., Tyagi, G., Jangir, D. K., Dawar, R. \& Gupta, N. Analysis of ovarian tumor pathology by Fourier Transform Infrared Spectroscopy. J Ovarian Res 3, 27 (2010).

58 Paraskevaidi, M. et al. Potential of mid-infrared spectroscopy as a non-invasive diagnostic test in urine for endometrial or ovarian cancer. Analyst (2018).

59 Taylor, S. E. et al. Infrared spectroscopy with multivariate analysis to interrogate endometrial tissue: a novel and objective diagnostic approach. Br J Cancer 104, 790-797 (2011).

60 Paraskevaidi, M. et al. Aluminium foil as an alternative substrate for the spectroscopic interrogation of endometrial cancer. J Biophotonics (2018).

61 Gajjar, K. et al. Histology verification demonstrates that biospectroscopy analysis of cervical cytology identifies underlying disease more accurately than conventional screening: removing the confounder of discordance. PLoS One 9, e82416 (2014).

62 Walsh, M. J. et al. IR microspectroscopy: potential applications in cervical cancer screening. Cancer Lett 246, 1-11 (2007).

63 Wood, B. R., Quinn, M. A., Burden, F. R. \& McNaughton, D. An investigation into FTIR spectroscopy as a biodiagnostic tool for cervical cancer. Biospectroscopy 2, 143-153 (1996).

64 Podshyvalov, A. et al. Distinction of cervical cancer biopsies by use of infrared microspectroscopy and probabilistic neural networks. Appl Opt 44, 3725-3734 (2005). 
1001

1002

1003

1004

1005

1006

1007

1008

1009

1010

1011

1012

1013

1014

1015

1016

1017

1018

1019

1020

1021

1022

1023

1024

1025

1026

1027

1028

1029

1030

1031

1032

1033

1034

1035

1036

1037

1038

65 Theophilou, G. et al. A biospectroscopic analysis of human prostate tissue obtained from different time periods points to a trans-generational alteration in spectral phenotype. Sci Rep 5, 13465 (2015).

66 Baker, M. J. et al. Investigating FTIR based histopathology for the diagnosis of prostate cancer. J Biophotonics 2 (2009).

67 Derenne, A., Gasper, R. \& Goormaghtigh, E. The FTIR spectrum of prostate cancer cells allows the classification of anticancer drugs according to their mode of action. Analyst 136 (2011).

68 Gazi, E. et al. A correlation of FTIR spectra derived from prostate cancer biopsies with Gleason grade and tumour stage. Eur Urol 50, 750-761 (2006).

69 Paraskevaidi, M. et al. Differential diagnosis of Alzheimer's disease using spectrochemical analysis of blood. Proc Natl Acad Sci USA, 201701517 (2017).

70 Carmona, P. et al. Discrimination analysis of blood plasma associated with Alzheimer's disease using vibrational spectroscopy. J Alzheimers Dis 34, 911-920 (2013).

71 Carmona, P., Molina, M., López-Tobar, E. \& Toledano, A. Vibrational spectroscopic analysis of peripheral blood plasma of patients with Alzheimer's disease. Anal Bioanal Chem 407, 7747-7756 (2015).

72 Paraskevaidi, M. et al. Blood-based near-infrared spectroscopy for the rapid low-cost detection of Alzheimer's disease. Analyst (2018).

73 Sitole, L., Steffens, F., Krüger, T. P. J. \& Meyer, D. Mid-ATR-FTIR Spectroscopic Profiling of HIV/AIDS Sera for Novel Systems Diagnostics in Global Health. OMICS 18, 513-523 (2014).

74 Coopman, R. et al. Glycation in human fingernail clippings using ATR-FTIR spectrometry, a new marker for the diagnosis and monitoring of diabetes mellitus. Clin Biochem 50, 62-67 (2017).

75 Scott, D. A. et al. Diabetes-related molecular signatures in infrared spectra of human saliva. Diabetol Metab Syndr 2, 48 (2010).

76 Varma, V. K., Kajdacsy-Balla, A., Akkina, S. K., Setty, S. \& Walsh, M. J. A label-free approach by infrared spectroscopic imaging for interrogating the biochemistry of diabetic nephropathy progression. Kidney Int 89, 1153-1159 (2016).

77 Lechowicz, L., Chrapek, M., Gaweda, J., Urbaniak, M. \& Konieczna, I. Use of Fouriertransform infrared spectroscopy in the diagnosis of rheumatoid arthritis: a pilot study. $\mathrm{Mol}$ Biol Rep 43, 1321-1326 (2016).

78 Canvin, J. et al. Infrared spectroscopy: shedding light on synovitis in patients with rheumatoid arthritis. Rheumatology 42, 76-82 (2003).

79 Oemrawsingh, R. M. et al. Near-infrared spectroscopy predicts cardiovascular outcome in patients with coronary artery disease. J Am Coll Cardiol 64, 2510-2518 (2014).

80 Wang, J. et al. Near-infrared spectroscopic characterization of human advanced atherosclerotic plaques. J Am Coll Cardiol 39, 1305-1313 (2002).

81 Martin, M. et al. The effect of common anticoagulants in detection and quantification of malaria parasitemia in human red blood cells by ATR-FTIR spectroscopy. Analyst (2017).

82 Khoshmanesh, A. et al. Detection and Quantification of Early-Stage Malaria Parasites in Laboratory Infected Erythrocytes by Attenuated Total Reflectance Infrared Spectroscopy and Multivariate Analysis. Anal Chem 86, 4379-4386 (2014).

83 Roy, S. et al. Simultaneous ATR-FTIR Based Determination of Malaria Parasitemia, Glucose and Urea in Whole Blood Dried onto a Glass Slide. Anal Chem 89, 5238-5245 (2017).

84 Markus, A. P. J. et al. New technique for diagnosis and monitoring of alcaptonuria: quantification of homogentisic acid in urine with mid-infrared spectrometry. Anal Chim Acta 429, 287-292 (2001).

85 Grimard, V. et al. Phosphorylation-induced Conformational Changes of Cystic Fibrosis Transmembrane Conductance Regulator Monitored by Attenuated Total Reflection-Fourier 
Transform IR Spectroscopy and Fluorescence Spectroscopy. J Biol Chem 279, 5528-5536 (2004).

86 Aksoy, C., Guliyev, A., Kilic, E., Uckan, D. \& Severcan, F. Bone marrow mesenchymal stem cells in patients with beta thalassemia major: molecular analysis with attenuated total reflection-Fourier transform infrared spectroscopy study as a novel method. Stem Cells Dev 21, 2000-2011 (2012).

87 Graça, G. et al. Mid-infrared (MIR) metabolic fingerprinting of amniotic fluid: A possible avenue for early diagnosis of prenatal disorders? Anal Chim Acta 764, 24-31 (2013).

88 Hasegawa, J. et al. Evaluation of placental function using near infrared spectroscopy during fetal growth restriction. J Perinatal Med 38, 29-32 (2010).

89 Theelen, T., Berendschot, T. T., Hoyng, C. B., Boon, C. J. \& Klevering, B. J. Near-infrared reflectance imaging of neovascular age-related macular degeneration. Graefe's Arch Clin Exp Ophthalmol 247, 1625 (2009).

90 Semoun, O. et al. Infrared features of classic choroidal neovascularisation in exudative agerelated macular degeneration. Br J Ophthalmol. 93, 182-185 (2009).

91 Peters, A. S. et al. Serum-infrared spectroscopy is suitable for diagnosis of atherosclerosis and its clinical manifestations. Vib Spectrosc 92, 20-26 (2017).

92 Afara, I. O., Prasadam, I., Arabshahi, Z., Xiao, Y. \& Oloyede, A. Monitoring osteoarthritis progression using near infrared (NIR) spectroscopy. Sci Rep 7, 11463 (2017).

$93 \mathrm{Bi}, \mathrm{X}$. et al. Fourier transform infrared imaging and MR microscopy studies detect compositional and structural changes in cartilage in a rabbit model of osteoarthritis. Anal Bioanal Chem 387, 1601-1612 (2007).

94 David-Vaudey, E. et al. Fourier Transform Infrared Imaging of focal lesions in human osteoarthritic cartilage. Eur Cell Mater 10, 60 (2005).

95 Trevisan, J., Angelov, P. P., Carmichael, P. L., Scott, A. D. \& Martin, F. L. Extracting biological information with computational analysis of Fourier-transform infrared (FTIR) biospectroscopy datasets: current practices to future perspectives. Analyst 137, 3202-3215 (2012).

96 Andrew Chan, K. L. \& Kazarian, S. G. Attenuated total reflection Fourier-transform infrared (ATR-FTIR) imaging of tissues and live cells. Chem Soc Rev 45, 1850-1864 (2016).

97 Pilling, M. \& Gardner, P. Fundamental developments in infrared spectroscopic imaging for biomedical applications. Chem Soc Rev 45, 1935-1957 (2016).

98 Martin, F. L. et al. Distinguishing cell types or populations based on the computational analysis of their infrared spectra. Nat Protoc 5, 1748-1760 (2010).

99 Butler, H. J. et al. Using Raman spectroscopy to characterize biological materials. Nat Protoc 11, 664-687 (2016).

100 Kong, L. et al. Characterization of bacterial spore germination using phase-contrast and fluorescence microscopy, Raman spectroscopy and optical tweezers. Nat Protoc 6, 625 (2011).

101 Harmsen, S., Wall, M. A., Huang, R. \& Kircher, M. F. Cancer imaging using surface-enhanced resonance Raman scattering nanoparticles. Nat Protoc 12, 1400 (2017).

102 Beckonert, O. et al. Metabolic profiling, metabolomic and metabonomic procedures for NMR spectroscopy of urine, plasma, serum and tissue extracts. Nat Protoc 2, 2692 (2007).

103 Felten, J. et al. Vibrational spectroscopic image analysis of biological material using multivariate curve resolution-alternating least squares (MCR-ALS). Nat Protoc 10, 217 (2015).

104 Yang, H., Yang, S., Kong, J., Dong, A. \& Yu, S. Obtaining information about protein secondary structures in aqueous solution using Fourier transform IR spectroscopy. Nat Protoc 10, 382 (2015).

105 Sreedhar, H. et al. High-definition Fourier transform infrared (FT-IR) spectroscopic imaging of human tissue sections towards improving pathology. J Vis Exp (2015). 
106 Varriale, A. et al. Fluorescence correlation spectroscopy assay for gliadin in food. Anal Chem 79, 4687-4689 (2007).

107 Song, X., Li, H., Al-Qadiri, H. M. \& Lin, M. Detection of herbicides in drinking water by surface-enhanced Raman spectroscopy coupled with gold nanostructures. J Food Meas Charact 7, 107-113 (2013).

108 Osborne, B. G. \& Fearn, T. Near-infrared spectroscopy in food analysis. Encyclopedia Anal Chem 5, 4069-4082 (2000).

109 Qu, J.-H. et al. Applications of near-infrared spectroscopy in food safety evaluation and control: A review of recent research advances. Crit Rev Food Sci Nut. 55, 1939-1954 (2015).

110 Penido, C. A. F., Pacheco, M. T. T., Lednev, I. K. \& Silveira, L. Raman spectroscopy in forensic analysis: identification of cocaine and other illegal drugs of abuse. J Raman Spectrosc 47, 2838 (2016).

111 Ryder, A. G. Classification of narcotics in solid mixtures using principal component analysis and Raman spectroscopy. J Forensic Sci 47, 275-284 (2002).

112 Harrigan, G. G. et al. Application of high-throughput Fourier-transform infrared spectroscopy in toxicology studies: contribution to a study on the development of an animal model for idiosyncratic toxicity. Toxicol Lett 146, 197-205 (2004).

113 Choo-Smith, L.-P. et al. Investigating microbial (micro) colony heterogeneity by vibrational spectroscopy. Appl Environ Microbiol 67, 1461-1469 (2001).

114 Helm, D., Labischinski, H., Schallehn, G. \& Naumann, D. Classification and identification of bacteria by Fourier-transform infrared spectroscopy. Microbiology 137, 69-79 (1991).

115 Carmona, P., Monzon, M., Monleon, E., Badiola, J. J. \& Monreal, J. In vivo detection of scrapie cases from blood by infrared spectroscopy. J Gen Virol 86, 3425-3431 (2005).

116 Cui, L. et al. A novel functional single-cell approach to probing nitrogen-fixing bacteria in soil communities by resonance Raman spectroscopy with 15N2 labelling. Anal Chem 10.1021/acs.analchem.7b05080. (2018).

117 Lasch, P. \& Naumann, D. Infrared spectroscopy in microbiology. Encyclopedia Anal Chem (2015).

118 Maquelin, K. et al. Identification of medically relevant microorganisms by vibrational spectroscopy. J Microbiol Methods 51, 255-271 (2002).

119 Day, J. S., Edwards, H. G., Dobrowski, S. A. \& Voice, A. M. The detection of drugs of abuse in fingerprints using Raman spectroscopy I: latent fingerprints. Spectrochim Acta A Mol Biomol Spectrosc 60, 563-568 (2004).

120 Macleod, N. A. \& Matousek, P. Emerging Non-invasive Raman Methods in Process Control and Forensic Applications. Pharm Res 25, 2205 (2008).

121 Lewis, I., Daniel Jr, N., Chaffin, N., Griffiths, P. \& Tungol, M. Raman spectroscopic studies of explosive materials: towards a fieldable explosives detector. Spectrochim Acta A 51, 19852000 (1995).

122 Hargreaves, M. D. \& Matousek, P. Threat detection of liquid explosive precursor mixtures by Spatially Offset Raman Spectroscopy (SORS). in Optics and photonics for counterterrorism and crime fighting V. Vol. 7486 74860B (International Society for Optics and Photonics).

123 Ali, E. M., Edwards, H. G., Hargreaves, M. D. \& Scowen, I. J. Raman spectroscopic investigation of cocaine hydrochloride on human nail in a forensic context. Anal Bioanal Chem 390, 1159-1166 (2008).

124 Vergote, G. J., Vervaet, C., Remon, J. P., Haemers, T. \& Verpoort, F. Near-infrared FT-Raman spectroscopy as a rapid analytical tool for the determination of diltiazem hydrochloride in tablets. Eur J Pharm Sci 16, 63-67 (2002).

125 Lohr, D. et al. Non-destructive determination of carbohydrate reserves in leaves of ornamental cuttings by near-infrared spectroscopy (NIRS) as a key indicator for quality assessments. Biosys Eng 158, 51-63 (2017). 
126 Heys, K. A., Shore, R. F., Pereira, M. G. \& Martin, F. L. Levels of Organochlorine Pesticides Are Associated with Amyloid Aggregation in Apex Avian Brains. Environ Sci Technol 51, 8672 8681 (2017).

127 Comino, F., Aranda, V., García-Ruiz, R. \& Domínguez-Vidal, A. Infrared spectroscopy as a tool for the assessment of soil biological quality in agricultural soils under contrasting management practices. Ecol Indicators 87, 117-126 (2018).

128 Eliasson, C., Macleod, N. \& Matousek, P. Noninvasive detection of concealed liquid explosives using Raman spectroscopy. Anal Chem 79, 8185-8189 (2007).

129 Liu, H.-B., Zhong, H., Karpowicz, N., Chen, Y. \& Zhang, X.-C. Terahertz spectroscopy and imaging for defense and security applications. Proc IEEE 95, 1514-1527 (2007).

130 Golightly, R. S., Doering, W. E. \& Natan, M. J. Surface-enhanced Raman spectroscopy and homeland security: a perfect match? ACS Nano 3, 2859-2869 (2009).

131 Sattlecker, M., Stone, N., Smith, J. \& Bessant, C. Assessment of robustness and transferability of classification models built for cancer diagnostics using Raman spectroscopy. J Raman Spectrosc 42, 897-903 (2011).

132 Isabelle, M. et al. Multi-centre Raman spectral mapping of oesophageal cancer tissues: a study to assess system transferability. Faraday Discuss 187, 87-103 (2016).

133 Guo, S. et al. Towards an improvement of model transferability for Raman spectroscopy in biological applications. Vib Spectrosc 91, 111-118 (2017).

134 Luo, X. et al. Calibration transfer across near infrared spectrometers for measuring hematocrit in the blood of grazing cattle. J Near Infrared Spec 25, 15-25 (2017).

135 Vaughan, A. A. et al. Liquid chromatography-mass spectrometry calibration transfer and metabolomics data fusion. Anal Chem 84, 9848-9857 (2012).

136 Rodriguez, J. D., Westenberger, B. J., Buhse, L. F. \& Kauffman, J. F. Standardization of Raman spectra for transfer of spectral libraries across different instruments. Analyst 136, 4232-4240 (2011).

137 de Andrade, E. W., de Lelis Medeiros de Morais, C., Lopes da Costa, F. S., de Lima, G. \& Michell, K. A Multivariate Control Chart Approach for Calibration Transfer between NIR Spectrometers for Simultaneous Determination of Rifampicin and Isoniazid in Pharmaceutical Formulation. Curr Anal Chem 14, 488-494 (2018).

138 Yu, B., Ji, H. \& Kang, Y. Standardization of near infrared spectra based on multi-task learning. Spectrosc Lett 49, 23-29 (2016).

$139 \mathrm{Ni}$, L., Han, M., Luan, S. \& Zhang, L. Screening wavelengths with consistent and stable signals to realize calibration model transfer of near infrared spectra. Spectrochim Acta A 206,350-8 (2019).

$140 \mathrm{Hu}, \mathrm{R}$. \& Xia, J. Calibration transfer of near infrared spectroscopy based on DS algorithm. in Electric Information and Control Engineering (ICEICE), 2011 International Conference on 3062-3065 (IEEE).

141 Forina, M. et al. Transfer of calibration function in near-infrared spectroscopy. Chemom Intellig Lab Syst 27, 189-203 (1995).

142 Xiao, H. et al. Comparison of benchtop Fourier-transform (FT) and portable grating scanning spectrometers for determination of total soluble solid contents in single grape berry (Vitis vinifera L.) and calibration transfer. Sensors 17, 2693 (2017).

143 Yahaya, O., MatJafri, M., Aziz, A. \& Omar, A. Visible spectroscopy calibration transfer model in determining $\mathrm{pH}$ of Sala mangoes. J Instrum 10, T05002 (2015).

144 Bin, J., Li, X., Fan, W., Zhou, J.-h. \& Wang, C.-w. Calibration transfer of near-infrared spectroscopy by canonical correlation analysis coupled with wavelet transform. Analyst 142, 2229-2238 (2017).

145 Monakhova, Y. B. \& Diehl, B. W. Transfer of multivariate regression models between high resolution NMR instruments: application to authenticity control of sunflower lecithin. Magn Reson Chem 54, 712-717 (2016). 
146 Zuo, Q., Xiong, S., Chen, Z.-P., Chen, Y. \& Yu, R.-Q. A novel calibration strategy based on background correction for quantitative circular dichroism spectroscopy. Talanta 174, 320324 (2017).

147 Koehler IV, F. W., Small, G. W., Combs, R. J., Knapp, R. B. \& Kroutil, R. T. Calibration transfer algorithm for automated qualitative analysis by passive Fourier transform infrared spectrometry. Anal Chem 72, 1690-1698 (2000).

148 Rodrigues, R. R. et al. Evaluation of calibration transfer methods using the ATR-FTIR technique to predict density of crude oil. Chemom Intellig Lab Syst 166, 7-13 (2017).

149 Wang, Y., Veltkamp, D. J. \& Kowalski, B. R. Multivariate instrument standardization. Anal Chem 63, 2750-2756 (1991).

150 Brouckaert, D., Uyttersprot, J.-S., Broeckx, W. \& De Beer, T. Calibration transfer of a Raman spectroscopic quantification method for the assessment of liquid detergent compositions from at-line laboratory to in-line industrial scale. Talanta 179, 386-392 (2018).

151 Andrade, E. V., Morais, C. d. L. M., Costa, F. S. L. \& Lima, K. M. G. A Multivariate Control Chart Approach for Calibration Transfer between NIR Spectrometers for Simultaneous Determination of Rifampicin and Isoniazid in Pharmaceutical Formulation. Curr Anal Chem 14, 1-7 (2018).

152 Zamora-Rojas, E., Pérez-Marín, D., De Pedro-Sanz, E., Guerrero-Ginel, J. \& Garrido-Varo, A. Handheld NIRS analysis for routine meat quality control: Database transfer from at-line instruments. Chemom Intellig Lab Syst 114, 30-35 (2012).

153 Panchuk, V., Kirsanov, D., Oleneva, E., Semenov, V. \& Legin, A. Calibration transfer between different analytical methods. Talanta 170, 457-463 (2017).

154 de Morais, C. d. L. M. \& de Lima, K. M. G. Determination and analytical validation of creatinine content in serum using image analysis by multivariate transfer calibration procedures. Anal Methods 7, 6904-6910 (2015).

155 Khaydukova, M. et al. Multivariate calibration transfer between two different types of multisensor systems. Sensors Actuators B Chem 246, 994-1000 (2017).

156 Barreiro, P. et al. Calibration Transfer Between Portable and Laboratory NIR Spectrophotometers. Acta Hortic (2008).

157 Sulub, Y., LoBrutto, R., Vivilecchia, R. \& Wabuyele, B. W. Content uniformity determination of pharmaceutical tablets using five near-infrared reflectance spectrometers: a process analytical technology (PAT) approach using robust multivariate calibration transfer algorithms. Anal Chim Acta 611, 143-150 (2008).

158 Zhang, L., Small, G. W. \& Arnold, M. A. Multivariate calibration standardization across instruments for the determination of glucose by Fourier transform near-infrared spectrometry. Anal Chem 75, 5905-5915 (2003).

159 Martens, H., Høy, M., Wise, B. M., Bro, R. \& Brockhoff, P. B. Pre - whitening of data by covariance - weighted pre - processing. J Chemom 17, 153-165 (2003).

160 Feudale, R. N. et al. Transfer of multivariate calibration models: a review. Chemom Intellig Lab Syst 64, 181-192 (2002).

161 Woody, N. A., Feudale, R. N., Myles, A. J. \& Brown, S. D. Transfer of multivariate calibrations between four near-infrared spectrometers using orthogonal signal correction. Anal Chem 76, 2595-2600 (2004).

162 Greensill, C., Wolfs, P., Spiegelman, C. \& Walsh, K. Calibration transfer between PDA-based NIR spectrometers in the NIR assessment of melon soluble solids content. Appl Spectrosc 55, 647-653 (2001).

163 Sjöblom, J., Svensson, O., Josefson, M., Kullberg, H. \& Wold, S. An evaluation of orthogonal signal correction applied to calibration transfer of near infrared spectra. Chemom Intellig Lab Syst 44, 229-244 (1998).

164 Andrews, D. T. \& Wentzell, P. D. Applications of maximum likelihood principal component analysis: incomplete data sets and calibration transfer. Anal Chim Acta 350, 341-352 (1997). 
165 Bouveresse, E., Massart, D. \& Dardenne, P. Calibration transfer across near-infrared spectrometric instruments using Shenk's algorithm: effects of different standardisation samples. Anal Chim Acta 297, 405-416 (1994).

166 Shenk, J. S. \& Westerhaus, M. O. Populations structuring of near infrared spectra and modified partial least squares regression. Crop Sci 31, 1548-1555 (1991).

167 Paatero, P. \& Tapper, U. Positive matrix factorization: A non - negative factor model with optimal utilization of error estimates of data values. Environmetrics 5, 111-126 (1994).

168 Xie, Y. \& Hopke, P. K. Calibration transfer as a data reconstruction problem. Anal Chim Acta 384, 193-205 (1999).

169 Goodacre, R. et al. On mass spectrometer instrument standardization and interlaboratory calibration transfer using neural networks. Anal Chim Acta 348, 511-532 (1997).

170 Chen, W.-R., Bin, J., Lu, H.-M., Zhang, Z.-M. \& Liang, Y.-Z. Calibration transfer via an extreme learning machine auto-encoder. Analyst 141, 1973-1980 (2016).

$171 \mathrm{Hu}, \mathrm{Y} .$, Peng, S., Bi, Y. \& Tang, L. Calibration transfer based on maximum margin criterion for qualitative analysis using Fourier transform infrared spectroscopy. Analyst 137, 5913-5918 (2012).

172 Fan, W., Liang, Y., Yuan, D. \& Wang, J. Calibration model transfer for near-infrared spectra based on canonical correlation analysis. Anal Chim Acta 623, 22-29 (2008).

173 Wang, Z., Dean, T. \& Kowalski, B. R. Additive background correction in multivariate instrument standardization. Anal Chem 67, 2379-2385 (1995).

174 Kennard, R. W. \& Stone, L. A. Computer Aided Design of Experiments. Technometrics 11, 137-148 (1969).

175 Palonpon, A. F. et al. Raman and SERS microscopy for molecular imaging of live cells. Nat Protoc 8, 677 (2013).

176 Witze, E. S., Old, W. M., Resing, K. A. \& Ahn, N. G. Mapping protein post-translational modifications with mass spectrometry. Nat Methods 4, 798 (2007).

177 Aebersold, R. \& Mann, M. Mass spectrometry-based proteomics. Nature 422, 198 (2003).

178 Pence, I. \& Mahadevan-Jansen, A. Clinical instrumentation and applications of Raman spectroscopy. Chem Soc Rev 45, 1958-1979 (2016).

179 Ibrahim, O. et al. Improved protocols for pre-processing Raman spectra of formalin fixed paraffin preserved tissue sections. Anal Methods 9, 4709-4717 (2017).

180 Tfayli, A. et al. Digital dewaxing of Raman signals: discrimination between nevi and melanoma spectra obtained from paraffin-embedded skin biopsies. Appl Spectrosc 63, 564570 (2009).

181 Byrne, H. J., Knief, P., Keating, M. E. \& Bonnier, F. Spectral pre and post processing for infrared and Raman spectroscopy of biological tissues and cells. Chem Soc Rev 45, 1865-1878 (2016).

182 Meade, A. D. et al. Studies of chemical fixation effects in human cell lines using Raman microspectroscopy. Anal Bioanal Chem 396, 1781-1791 (2010).

183 Baker, M. J. et al. Developing and understanding biofluid vibrational spectroscopy: a critical review. Chem Soc Rev 45, 1803-1818 (2016).

184 Bonifacio, A., Cervo, S. \& Sergo, V. Label-free surface-enhanced Raman spectroscopy of biofluids: fundamental aspects and diagnostic applications. Anal Bioanal Chem 407, 82658277 (2015).

185 Mitchell, A. L., Gajjar, K. B., Theophilou, G., Martin, F. L. \& Martin-Hirsch, P. L. Vibrational spectroscopy of biofluids for disease screening or diagnosis: translation from the laboratory to a clinical setting. J Biophotonics 7, 153-165 (2014).

186 Lovergne, L. et al. Biofluid infrared spectro-diagnostics: pre-analytical considerations for clinical applications. Faraday Discuss 187, 521-537 (2016).

187 Bonifacio, A. et al. Surface-enhanced Raman spectroscopy of blood plasma and serum using Ag and Au nanoparticles: a systematic study. Anal Bioanal Chem 406, 2355-2365 (2014). 
188 Paraskevaidi, M., Martin-Hirsch, P. L. \& Martin, F. L. ATR-FTIR Spectroscopy Tools for Medical Diagnosis and Disease Investigation. In Nanotechnology Characterization Tools for Biosensing and Medical Diagnosis, Springer, Berlin, Heidelberg, 163-211 (2017).

189 Mitchell, B. L., Yasui, Y., Li, C. I., Fitzpatrick, A. L. \& Lampe, P. D. Impact of freeze-thaw cycles and storage time on plasma samples used in mass spectrometry based biomarker discovery projects. Cancer Inform 1 (2005).

190 Glassford, S. E., Byrne, B. \& Kazarian, S. G. Recent applications of ATR FTIR spectroscopy and imaging to proteins. Biochim Biophys Acta 1834, 2849-2858 (2013).

191 Kundu, J., Le, F., Nordlander, P. \& Halas, N. J. Surface enhanced infrared absorption (SEIRA) spectroscopy on nanoshell aggregate substrates. Chem Phys Lett 452, 115-119 (2008).

192 Jones, S., Carley, S. \& Harrison, M. An introduction to power and sample size estimation. Emerg Med J 20, 453-458 (2003).

193 Beebe, K. R., Pell, R. J. \& Seasholtz, M. B. Chemometrics: a practical guide. In Wiley New York 4 (1998).

194 Pavia, D. L., Lampman, G. M., Kriz, G. S. \& Vyvyan, J. A. Introduction to spectroscopy. In Cengage Learning (2008).

195 Savitzky, A. \& Golay, M. J. Smoothing and differentiation of data by simplified least squares procedures. Anal Chem 36, 1627-1639 (1964).

196 Geladi, P., MacDougall, D. \& Martens, H. Linearization and scatter-correction for nearinfrared reflectance spectra of meat. Appl Spectrosc 39, 491-500 (1985).

197 Barnes, R., Dhanoa, M. S. \& Lister, S. J. Standard normal variate transformation and detrending of near-infrared diffuse reflectance spectra. Appl Spectrosc 43, $772-777$ (1989).

198 Brereton, R. G. Chemometrics: data analysis for the laboratory and chemical plant. In John Wiley \& Sons (2003).

199 Hastie, T., Tibshirani, R. \& Friedman, J. The elements of statistical learning 2nd edition. In New York: Springer (2009).

200 Bro, R. \& Smilde, A. K. Principal component analysis. Anal Methods 6, 2812-2831 (2014).

201 Martin, F. L. et al. Identifying variables responsible for clustering in discriminant analysis of data from infrared microspectroscopy of a biological sample. J Comput Biol 14, 1176-1184 (2007).

202 Martens, H. \& Martens, M. Modified Jack-knife estimation of parameter uncertainty in bilinear modelling by partial least squares regression (PLSR). Food Qual Prefer 11, 5-16 (2000).

203 Rousseeuw, P. J. \& Hubert, M. Robust statistics for outlier detection. Wiley Interdiscip Rev Data Min Knowl Discov 1, 73-79 (2011).

204 Jiang, F., Liu, G., Du, J. \& Sui, Y. Initialization of K-modes clustering using outlier detection techniques. Inf Sci 332, 167-183 (2016).

205 Domingues, R., Filippone, M., Michiardi, P. \& Zouaoui, J. A comparative evaluation of outlier detection algorithms: Experiments and analyses. Pattern Recognit 74, 406-421 (2018).

206 Bakeev, K. A. Process analytical technology: spectroscopic tools and implementation strategies for the chemical and pharmaceutical industries. In John Wiley \& Sons (2010).

207 Kuligowski, J., Quintás, G., Herwig, C. \& Lendl, B. A rapid method for the differentiation of yeast cells grown under carbon and nitrogen-limited conditions by means of partial least squares discriminant analysis employing infrared micro-spectroscopic data of entire yeast cells. Talanta 99, 566-573 (2012).

208 Morais, C. L. \& Lima, K. M. Comparing unfolded and two-dimensional discriminant analysis and support vector machines for classification of EEM data. Chemom Intell Lab Syst 170, 1-2 (2017).

209 Dixon, S. J. \& Brereton, R. G. Comparison of performance of five common classifiers represented as boundary methods: Euclidean Distance to Centroids, Linear Discriminant 
Analysis, Quadratic Discriminant Analysis, Learning Vector Quantization and Support Vector Machines, as dependent on data structure. Chemom Intell Lab Syst 95, 1-17 (2009).

210 Brereton, R. G. \& Lloyd, G. R. Partial least squares discriminant analysis: taking the magic away. J Chemom 28, 213-225 (2014).

211 Cover, T. \& Hart, P. Nearest neighbor pattern classification. IEEE Trans Inf Theory 13, 21-27 (1967).

212 Cortes, C. \& Vapnik, V. Support-vector networks. Mach Learn 20, 273-297 (1995).

213 Abraham, A. Artificial neural networks. handbook of measuring system design (2005).

214 Fawagreh, K., Gaber, M. M. \& Elyan, E. Random forests: from early developments to recent advancements. Systems Science \& Control Engineering 2, 602-609 (2014).

215 LeCun, Y., Bengio, Y. \& Hinton, G. Deep learning. Nature 521, 436 (2015).

216 Seasholtz, M. B. \& Kowalski, B. The parsimony principle applied to multivariate calibration. Anal Chim Acta 277, 165-177 (1993).

217 Morais, C. L. \& Lima, K. M. Principal Component Analysis with Linear and Quadratic Discriminant Analysis for Identification of Cancer Samples Based on Mass Spectrometry. J Braz Chem Soc, 31 (2017).

218 Hibbert, D. B. Vocabulary of concepts and terms in chemometrics (IUPAC Recommendations 2016). Pure Appl Chem 88, 407-443 (2016).

219 McCall, J. Genetic algorithms for modelling and optimisation. J Comput Appl Math 184, 205222 (2005).

220 Soares, S. F. C., Gomes, A. A., Araujo, M. C. U., Galvão Filho, A. R. \& Galvão, R. K. H. The successive projections algorithm. Trends Anal Chem 42, 84-98 (2013).

221 Kamandar, M. \& Ghassemian, H. Maximum relevance, minimum redundancy feature extraction for hyperspectral images. In Electrical Engineering (ICEE), 2010 18th Iranian Conference on. 254-259 (IEEE). 
Figure 1. IR spectra of healthy control (absence of disease) samples varying ATR-FTIR instruments and operators. Average (a) raw and (b) pre-processed IR spectra for healthy control samples measured across three different ATR-FTIR spectrometers in the same institute (A, B and C). Average (c) raw and (d) pre-processed IR spectra for healthy control samples across two different operators (Operator 1 and 2).

Figure 2. PCA scores for healthy control (absence of disease) samples varying ATR-FTIR instruments before and after standardization. (a) PCA scores for healthy control samples across three different ATR-FTIR spectrometers in the same institute (A, B and C) after preprocessing but before PDS; (b) PCA scores for healthy control samples across three different

ATR-FTIR spectrometers in the same institute (A, B and C) after PDS (model built with 55 transfer samples and window size of 23 wavenumbers). The dotted blue circle shows $95 \%$ confidence ellipse (two-sided). Each measurement observation (circle) corresponds to the data acquired from a unique operator.

Figure 3. Flowchart for standardization using Direct Standardization (DS).

Figure 4. Flowchart for a standardization protocol using different experimental conditions.

Figure 5. Discriminant function (DF) plots using PCA-LDA to discriminate healthy control (absence of disease) samples from ovarian cancer samples varying the instrument. (a) DF plot of the PCA-LDA model for the primary system; (b) DF plot of the PCA-LDA model for the primary system predicting the samples from the secondary systems. Sample index represents the number of samples' spectra.

Figure 6. PCA-LDA results for DS and PDS standardisation models for spectra collected by the three different instruments. (a) Misclassification rate in $\%$ for the validation set of the secondary system varying the number of transfer samples in \% from the primary system for 
DS optimization; (b) DF plot of the PCA-LDA model for the primary system predicting the

1396 validation set from the secondary system after DS; (c) Misclassification rate in \% for the validation set of the secondary system varying the window size for PDS optimization; (d) DF plot of the PCA-LDA model for the primary system predicting the validation set from the secondary system after PDS. Transfer samples (\%) refer to the percentage of training samples' spectra from the primary instrument that are used to transform the signal obtained using the secondary instrument.

Figure 7. Discriminant function (DF) plots using PCA-LDA to discriminate healthy control (absence of disease) samples from ovarian cancer samples varying the operator. (a) DF plot of the PCA-LDA model for the primary system (Operator 1); (b) DF plot of the PCA-LDA model for the primary system predicting the samples from the secondary system (Operator 2).

Figure 8. PCA-LDA results for DS and PDS standardisation models for spectra collected by two different operators. (a) Misclassification rate in $\%$ for the validation set of the secondary system (Operator 2) varying the number of transfer samples in $\%$ from the primary system (Operator 1) for DS optimization; (b) DF plot of the PCA-LDA model for the primary system predicting the validation set from the secondary system after DS; (c) Misclassification rate in $\%$ for the validation set of the secondary system varying the window size for PDS optimization; (d) DF plot of the PCA-LDA model for the primary system predicting the validation set from the secondary system after PDS. 